JOURNAL OF THE AMERICAN MATHEMATICAL SOCIETY

Volume 21, Number 1, January 2008, Pages 259-281

S 0894-0347(06)00553-4

Article electronically published on October 18, 2006

\title{
SCHUBERT POLYNOMIALS FOR THE AFFINE GRASSMANNIAN
}

THOMAS LAM

\section{INTRODUCTION}

Let $G$ be a complex simply connected simple group and $K$ a maximal compact subgroup. Let $\mathbf{F}=\mathbb{C}((t))$ denote the field of formal Laurent series and $\mathbf{O}=\mathbb{C}[[t]]$ the ring of formal power series. The quotient $\mathcal{G} r=G(\mathbf{F}) / G(\mathbf{O})$ is known as the affine Grassmannian of $G$. It is known that $\mathcal{G} r$ is homotopy equivalent to the space of based loops $\Omega K$ into the compact group $K$ (see [9, 30]). The multiplication on $\Omega K$ endows the homology $H_{*}(\mathcal{G} r)$ and cohomology $H^{*}(\mathcal{G} r)$ with structures of dual Hopf algebras. These Hopf algebras were first identified by Bott [4] (another description is given by Ginzburg [10, which we shall not use). The Bruhat decomposition of $G(\mathbf{F})$ induces a stratification of $\mathcal{G} r$ by Schubert cells $\Omega_{w}$, indexed by Grassmannian elements $w$ of the affine Weyl group $W_{\text {aff. }}$. We denote by $\sigma_{w} \in H_{*}(\mathcal{G} r)$ and $\sigma^{w} \in H^{*}(\mathcal{G} r)$ the Schubert classes in homology and cohomology.

When $G=S L(n, \mathbb{C})$, Bott identifies $H_{*}(\mathcal{G} r)$ and $H^{*}(\mathcal{G} r)$ with a subring $\Lambda_{n}$ and a quotient $\Lambda^{n}$ of the ring of symmetric functions. Our main theorem (Theorem 7.1) identifies the Schubert classes $\sigma_{w} \in H_{*}(\mathcal{G} r)$ and $\sigma^{w} \in H^{*}(\mathcal{G} r)$ as explicit symmetric functions under this isomorphism. These symmetric functions are combinatorially defined: in homology the $\sigma_{w} \in H_{*}(\mathcal{G} r)$ are represented by Lascoux-LapointeMorse's $k$-Schur functions $s_{w}^{(k)}(x) \in \Lambda_{n}$ and in cohomology the $\sigma^{w} \in H^{*}(\mathcal{G} r)$ are represented by the dual $k$-Schur functions (or affine Schur functions) $\tilde{F}_{w}(x) \in \Lambda^{n}$; see [21, 24, 17. Our theorem was originally conjectured by Mark Shimozono (the conjecture was made explicit in the cohomology case by Jennifer Morse).

Kostant and Kumar [14 studied the topology of homogeneous spaces for arbitrary Kac-Moody groups and in particular calculated the structure constants of $H^{*}(\mathcal{G} r)$ in the Schubert basis, using the algebraic construction of the nilHecke ring. Our connection between the topology and the combinatorics proceeds via the study of three subalgebras of the nilHecke ring. The first subalgebra is the nilCoxeter algebra $\mathbb{A}_{0}$, which is the algebra generated by the "divided difference" operators. The second algebra is a certain centralizer algebra $Z_{\mathbb{A}_{\text {aff }}}(S)$ which we call the Peterson subalgebra. Peterson 28 constructs an isomorphism $j$ from the $T$-equivariant homology $H_{T}(\mathcal{G} r)$ of the affine Grassmannian to $Z_{\mathbb{A}_{\mathrm{aff}}}(S)$. The third algebra $\mathbb{B}$ is a combinatorially defined subalgebra of $\mathbb{A}_{0}$, which we call the (affine) Fomin-Stanley

Received by the editors April 7, 2006.

2000 Mathematics Subject Classification. Primary 05E05; Secondary 14N15.

Key words and phrases. Schubert polynomials, symmetric functions, Schubert calculus, affine Grassmannian.

(C)2006 American Mathematical Society Reverts to public domain 28 years from publication 
subalgebra. It is (in the finite case) implicitly used in the construction of Schubert polynomials and Stanley symmetric functions by Fomin and Stanley [7].

These algebras are tied together by the study of elements $s_{w}^{(k)} \in \mathbb{B}$, introduced in [17, called non-commutative $k$-Schur functions. We show that they have a trio of descriptions:

- they are images of the $k$-Schur functions under an explicit isomorphism between symmetric functions and the Fomin-Stanley subalgebra;

- they are characterized by a leading "Grassmannian term" and a certain commutation property with the scalars $S=H^{T}(\mathrm{pt})$; and

- they are the evaluation at 0 of the images of the equivariant homology Schubert classes $\sigma_{(w)} \in H_{T}(\mathcal{G} r)$ under Peterson's map $j$.

The three descriptions establish the connection between the topology, algebra and combinatorics.

As a corollary of our main result, we establish conjectured positivity properties of $k$-Schur functions and affine Schur functions, via geometric positivity results of Graham, Kumar and Peterson [11, 16, 28]. In the other direction, certain conjectured combinatorial properties of $k$-Schur functions suggest interesting geometric properties, the most striking of which is the connection with Macdonald polynomials. Our results also gives new understanding to properties of symmetric functions. For example, the Hall inner product is given an interpretation as a pairing between homology and cohomology; Stanley symmetric functions are given a direct geometric interpretation (Remark 8.6) and their symmetry is explained by the commutativity of $H_{*}(\mathcal{G} r)$. We recover the Schur-positivity of Stanley symmetric functions [5, 26] and prove the more general (affine Schur-)positivity for affine Stanley symmetric functions conjectured in 17 .

Together with Lapointe, Morse and Shimozono [19], we develop a combinatorial framework for $k$-Schur functions and affine Schur functions suitable for the study of the affine Grassmannian. In particular, we obtain affine Pieri rules for $H_{*}(\mathcal{G} r)$ and $H^{*}(\mathcal{G} r)$.

Some of our results generalize to all simple Lie types, with the exception of the combinatorics. We hope to rectify this in a later work.

Organization. In Sections 2 5, we will work in arbitrary Lie type. In Section 2 , we establish basic notation and facts about the affine Weyl group and equivariant (co)homology of the affine Grassmannian; we work only in the case of a simple and simply-connected group. In Section 3, we introduce Kostant-Kumar's nilHecke ring and state the main theorem connecting it with the topology. In Section 4, we give a short but hopefully sufficiently detailed exposition of Peterson's $j$-homomorphism. Since Peterson's work 28, has yet to be published, we give more explanations here than just those needed to state Peterson's result. However, we do not aim to give a systematic development of Peterson's construction. In Section 5. we study a subalgebra of the nilHecke ring which we call the affine Fomin-Stanley subalgebra. We show that it is a model for the homology $H_{*}(\mathcal{G} r)$.

From Section 6, onwards, we specialize to type $A$. In Section 6, we establish notation for symmetric functions and, following [17, define affine Stanley symmetric functions, $k$-Schur functions and non-commutative $k$-Schur functions. In Section 7 , we state and prove our main theorem. The methods used here are essentially combinatorial. In Section 8, we explain a number of positivity properties. In 
Section 9 we give directions for further study and also identify and explain the center of the affine nilCoxeter algebra.

A preliminary shortened version of this work appeared as [18]. We should caution that some of the notation has changed. In particular $W$ now refers to the finite Weyl group and $W_{\text {aff }}$ refers to the affine Weyl group.

\section{EQUiVARIANT HOMOLOGY AND COHOMOLOGY of the AFFine Grassmannian}

More details for the material in this section can be found in [16, 13.

2.1. Affine Weyl group. Let $W$ be a crystallographic Coxeter group and let $\left\{r_{i} \mid i \in I\right\}$ denote its simple generators. Let $R$ be the root system for $W$. Let $R^{+}$ denote the positive roots, $R^{-}$the negative roots and $\left\{\alpha_{i} \mid i \in I\right\}$ the simple roots. Let $Q=\bigoplus_{i \in I} \alpha_{i}$ denote the root lattice and let $Q^{\vee}=\bigoplus_{i \in I} \alpha_{i}^{\vee}$ denote the co-root lattice. Let $h_{\mathbb{Z}}^{*}$ and $h_{\mathbb{Z}}$ denote a weight lattice and co-weight lattice, respectively. We assume that maps $Q \rightarrow h_{\mathbb{Z}}^{*}$ and $Q^{\vee} \rightarrow h_{\mathbb{Z}}$ are given and fixed. Let $\langle.,$.$\rangle denote$ the pairing between $h_{\mathbb{Z}}$ and $h_{\mathbb{Z}}^{*}$.

Let $W_{\text {aff }}=W \ltimes Q^{\vee}$ denote the affine Weyl group. Let $r_{0}$ denote the additional simple generator of $W_{\text {aff. }}$. For an element $\lambda \in Q^{\vee}$, we let $t_{\lambda}$ denote the corresponding translation element in $W_{\text {aff }}$. Note that translations are written multiplicatively: $t_{\lambda} \cdot t_{\mu}=t_{\lambda+\mu}$. We have the conjugation formula $w t_{\lambda} w^{-1}=t_{w \cdot \lambda}$. For a real root $\alpha$ of the affine root system $Q_{\mathrm{aff}}=\bigoplus_{i \in I \cup\{0\}} \alpha_{i}$, we let $r_{\alpha}$ denote the corresponding reflection.

Let $\ell: W_{\text {aff }} \rightarrow \mathbb{N}$ denote the length function of $W_{\text {aff }}$. We note the following formula for the length of the element $w t_{\lambda} \in W_{\text {aff }}$ (where $w \in W$ and $\lambda \in Q^{\vee}$ ):

$$
\ell\left(w t_{\lambda}\right)=\sum_{\alpha \in R^{+}}|\langle\lambda, \alpha\rangle+\chi(w \cdot \alpha)|
$$

where $\chi(\alpha)=0$ if $\alpha \in R^{+}$and $\chi(\alpha)=1$ otherwise.

Let $W^{0}$ denote the minimal length coset representatives of $W_{\text {aff }} / W$, which we call Grassmannian elements. There is a natural bijection between $W^{0}$ and $Q^{\vee}$ : each coset $W_{\text {aff }} / W$ contains one element from each set. Using the length formula, one can show that $W^{0} \cap Q^{\vee}=Q^{-}$, the (translations corresponding to) elements of the co-root lattice which are anti-dominant. In fact an element $w t_{\lambda}$ lies in $W^{0}$ if and only if $t_{\lambda} \in Q^{-}$and $w \in W^{\lambda}$ where $W^{\lambda}$ is the set of minimal length representatives of $W / W_{\lambda}$ and $W_{\lambda}$ is the stabilizer subgroup of $\lambda$.

2.2. Affine Grassmannian. Let $G$ be a simple and simply-connected complex algebraic group with Weyl group $W$. Let $K$ denote a maximal compact subgroup and $T$ a maximal torus in $K$. Let $h_{\mathbb{Z}}^{\vee}$ be the weight lattice and $h_{\mathbb{Z}}$ the co-weight lattice of $T$. Since $G$ is simply connected, $Q^{\vee} \hookrightarrow h_{\mathbb{Z}}$ is an isomorphism.

Let $\mathbf{F}=\mathbb{C}((t))$ and $\mathbf{O}=\mathbb{C}[[t]]$. The affine Grassmannian $\mathcal{G} r=\mathcal{G} r_{G}$ is the ind-scheme $G(\mathbf{F}) / G(\mathbf{O})$ (see [16] for more on the ind-scheme structure). It is a homogeneous space for the affine Kac-Moody group $\mathcal{G}$ associated to $W_{\text {aff }}$, which for our purposes can be taken simply to be $\mathcal{G}=G(\mathbf{F})$. The space $\mathcal{G} r$ is homotopyequivalent to the space $\Omega K$ of based loops in $K$; see [9, 30]. Since $K$ is simplyconnected, $\mathcal{G} r$ is connected. Each co-character $\lambda: S^{1} \rightarrow T \in Q^{\vee}$ gives a point in $\Omega K$, which we denote $t_{\lambda}$. These are $T$-fixed points of $\Omega K$. We let $S=S\left(h_{\mathbb{Z}}^{*}\right)=$ $H^{T}(\mathrm{pt})$ denote the symmetric algebra of $h_{\mathbb{Z}}^{*}$. Since $T$ does not contain the rotation 
action of $S^{1}$ on $\Omega K$, the image of the imaginary root $\delta=\alpha_{0}+\theta$ in $h_{\mathbb{Z}}^{*}$ is 0 . In other words, the map $Q_{\text {aff }} \rightarrow h_{\mathbb{Z}}^{*}$ is given by $\alpha_{0} \mapsto-\theta$ where $\theta$ is the highest root of the root system $R$.

The group $\mathcal{G}$ possesses a Bruhat decomposition $\mathcal{G}=\bigcup_{w \in W_{\text {aff }}} \mathcal{B} w \mathcal{B}$ where $\mathcal{B}$ denotes the Iwahori subgroup. The Bruhat decomposition induces a decomposition of $\mathcal{G} r$ into Schubert cells $\Omega_{w}=\mathcal{B} w G(\mathbf{O}) \subset G(\mathbf{F}) / G(\mathbf{O})$ :

$$
\mathcal{G} r=\bigsqcup_{w \in W^{0}} \Omega_{w}=\bigcup_{w \in W^{0}} X_{w}
$$

where the Schubert varieties $X_{w}$ are the closures of $\Omega_{w}$. In this paper we will be concerned with the homology $H_{*}(\mathcal{G} r)$, cohomology $H^{*}(\mathcal{G} r)$, torus-equivariant homology $H_{T}(\mathcal{G} r)$ and torus-equivariant cohomology $H^{T}(\mathcal{G} r)$ of the affine Grassmannian. Note that the torus $T$ acts on $\Omega K$ by (pointwise) conjugation. We will denote the Schubert classes in homology, cohomology, equivariant homology and equivariant cohomology as follows (see [16] for relevant definitions)

$$
\sigma_{w} \in H_{*}(\mathcal{G} r), \sigma^{w} \in H^{*}(\mathcal{G} r), \sigma_{(w)} \in H_{T}(\mathcal{G} r), \sigma^{(w)} \in H^{T}(\mathcal{G} r) .
$$

More generally, we will use the notation

$$
\sigma_{w}^{\mathcal{P}} \in H_{*}(\mathcal{G} / \mathcal{P}), \sigma_{(w)}^{\mathcal{P}} \in H_{T}(\mathcal{G} / \mathcal{P}), \sigma_{\mathcal{P}}^{w} \in H^{*}(\mathcal{G} / \mathcal{P}), \sigma_{\mathcal{P}}^{(w)} \in H^{T}(\mathcal{G} / \mathcal{P})
$$

for the Schubert classes for $\mathcal{G} / \mathcal{P}$ where $\mathcal{P} \subset \mathcal{G}$ is a parabolic subgroup. Throughout this paper, all homology and cohomology rings will be with $\mathbb{Z}$-coefficients. We should note that the equivariant homology theory that we use is equivariant BorelMoore homology. Since the stratification by Schubert cells is equivariant formal for the $T$-action, we may think of $H_{T}(\mathcal{G} r)$ as the subspace of $\operatorname{Hom}_{S}\left(H^{T}(\mathcal{G} r), S\right)$ with basis $\sigma_{(w)}$ dual to $\sigma^{(w)}$; see for example [11. (in particular Section 4.3) for the construction of these classes, which we omit. Both $H_{T}(\mathcal{G} r)$ and $H^{T}(\mathcal{G} r)$ are free modules over $S=H^{T}(\mathrm{pt})$ with basis the Schubert classes.

The main aim of this article is the identification of the classes $\sigma_{w}$ and $\sigma^{w}$ explicitly, as polynomials. These polynomials can be considered affine analogues of Lascoux and Schützenberger's Schubert polynomials [25], which are polynomial representatives for cohomology Schubert classes of the finite flag variety $K / T$. Note that there does not seem to be a classical, finite analogue of the homology representatives $\sigma_{w}$, though in special cases the homology polynomial representatives turn out to be Schur functions.

\section{Kostant-Kumar's NILHECKe RING}

The discussion of this section works for symmetrizable Kac-Moody groups, but we will restrict our notation to the affine case and make a small modification to the general theory (Remark 3.2). See [16, 28, for further details.

3.1. The affine nilHecke ring. Let $\mathbb{A}_{\text {aff }}$ denote the affine nilHecke ring of Kostant and Kumar. (Note that Kostant and Kumar define the nilHecke ring over the rationals, but we have found it more convenient, following Peterson 28], to work over $\mathbb{Z}$.) It is the ring with a 1 given by generators $\left\{A_{i} \mid i \in I \cup\{0\}\right\} \cup\left\{\lambda \mid \lambda \in h_{\mathbb{Z}}^{*}\right\}$ 
and the relations

$$
\begin{aligned}
A_{i} \lambda & =\left(r_{i} \cdot \lambda\right) A_{i}+\left\langle\lambda, \alpha_{i}^{\vee}\right\rangle \cdot 1 & & \text { for } \lambda \in h_{\mathbb{Z}}^{*}, \\
A_{i} A_{i} & =0, & & \\
\left(A_{i} A_{j}\right)^{m} & =\left(A_{j} A_{i}\right)^{m} & & \text { if }\left(r_{i} r_{j}\right)^{m}=\left(r_{j} r_{i}\right)^{m},
\end{aligned}
$$

where the "scalars" $\lambda \in h_{\mathbb{Z}}^{*}$ commute with other scalars. The finite nilHecke ring is the subring of $\mathbb{A}_{\text {aff }}$ generated by $\left\{A_{i} \mid i \in I\right\} \cup\left\{\lambda \mid \lambda \in h_{\mathbb{Z}}^{*}\right\}$. Combinatorially, one should think of the elements $A_{i}$ as divided difference operators.

Let $w \in W_{\text {aff }}$ and let $w=r_{i_{1}} \cdots r_{i_{l}}$ be a reduced decomposition of $w$. Then $A_{w}:=A_{i_{1}} \cdots A_{i_{l}}$ is a well-defined element of $\mathbb{A}_{\mathrm{aff}}$, where $A_{\text {id }}=1$. By 14, Theorem 4.6], the $\operatorname{set}\left\{A_{w} \mid w \in W_{\text {aff }}\right\}$ is an $S$-basis of $\mathbb{A}_{\text {aff }}$, where $S=S\left(h_{\mathbb{Z}}^{*}\right)$ denotes the symmetric algebra of $h_{\mathbb{Z}}^{*}$ as in Section 2 The map $W_{\text {aff }} \mapsto \mathbb{A}_{\text {aff }}$ given by $r_{i} \mapsto 1-\alpha_{i} A_{i} \in \mathbb{A}_{\text {aff }}$ is a homomorphism. Abusing notation, we write $w \in \mathbb{A}_{\text {aff }}$ for the element in the nilHecke ring corresponding to $w \in W_{\text {aff }}$ under this map. Then $W_{\text {aff }}$ is a basis for $\mathbb{A}_{\text {aff }}$ over $\operatorname{Frac}(S)$ (not over $S$ since $A_{i}=\frac{1}{\alpha_{i}}\left(1-r_{i}\right)$ ).

For later use, we note the following straightforward result, whose proof we omit; see [14, Proposition 4.30].

Lemma 3.1. Let $w \in W_{\mathrm{aff}}$ and $\lambda \in S$ be of degree 1. Then

$$
A_{w} \lambda=(w \cdot \lambda) A_{w}+\sum_{w r_{\alpha} \ll w}\left\langle\lambda, \alpha^{\vee}\right\rangle A_{w r_{\alpha}},
$$

where $\alpha$ is always taken to be a positive root of $W_{\mathrm{aff}}$. Here $\lessdot$ denotes a cover in strong Bruhat order.

The coefficients $\left\langle\lambda, \alpha^{\vee}\right\rangle$ are known as Chevalley coefficients.

Remark 3.2. The affine nilHecke ring $\mathbb{A}_{\mathrm{aff}}$ defined above is slightly different from the nilHecke ring $\mathbb{A}(\hat{R})$ associated to the affine root system $\hat{R}$ of $W_{\text {aff }}$, as defined in 14. Most importantly in our setup, the image of the imaginary root $\delta=\alpha_{0}+\theta$ in $h_{\mathbb{Z}}^{*}$ is 0 . In other words, we do not consider the additional $S^{1}$-equivariance obtained by rotating the loops in $\Omega K$. With this additional equivariance, the equivariant homology $H_{T \times S^{1}}(\mathcal{G} r)$ is actually non-commutative (compare with 3 ). Another difference is that the center of $\mathbb{A}(\hat{R})$ is much smaller than that of $\mathbb{A}_{\text {aff }}$. Shrawan Kumar pointed out to us that the center of $\mathbb{A}(\hat{R})$ is just the Weyl-group invariants of the scalars. We shall later find explicitly non-trivial elements in the center of $\mathbb{A}_{\text {aff }}$; see Section 9 .

3.2. Action on the cohomology ring $H^{T}(\mathcal{G} / \mathcal{P})$. The ring $\mathbb{A}_{\text {aff }}$ acts as generalized BGG-Demazure operators on $H^{T}(X)$ for any $L K$-space $X$ (here $L K$ is the space of all continuous loops into $K$ ). The element $A_{i}$ corresponds to the map $H^{*}(\mathcal{G} / \mathcal{B}) \rightarrow H^{*-2}(\mathcal{G} / \mathcal{B})$ obtained by integration along the fibers of the $\mathbb{P}^{1}$-fibration $\mathcal{G} / \mathcal{B} \rightarrow \mathcal{G} / \mathcal{P}_{i}$ where the $\mathcal{P}_{i}$ are the minimal parabolic subgroups; see [2, 16].

Fix $\mathcal{P}$ a parabolic subgroup of $\mathcal{G}$ and let $W_{\text {aff }}^{P}$ be the minimal length coset representatives for the quotient of $W_{\text {aff }}$ by the corresponding parabolic subgroup $W_{P} \subset$ $W_{\text {aff }}$. Then in terms of the Schubert classes $\sigma_{\mathcal{P}}^{(w)} \in H^{T}(\mathcal{G} / \mathcal{P})$ and $\sigma_{(w)}^{\mathcal{P}} \in H_{T}(\mathcal{G} / \mathcal{P})$, where $w \in W_{\text {aff }}^{P}$ we have

$$
A_{v} \cdot \sigma_{\mathcal{P}}^{(w)}= \begin{cases}(-1)^{\ell(v)} \sigma_{\mathcal{P}}^{(v w)} & \text { if } \ell\left(v^{-1}\right)+\ell(v w)=\ell(w) \text { and } v w \in W_{\text {aff }}^{\mathcal{P}}, \\ 0 & \text { otherwise }\end{cases}
$$


and

$$
A_{v} \cdot \sigma_{(w)}^{\mathcal{P}}= \begin{cases}\sigma_{(v w)}^{\mathcal{P}} & \text { if } \ell(v w)=\ell(v)+\ell(w) \text { and } v w \in W_{\mathrm{aff}}^{P}, \\ 0 & \text { otherwise. }\end{cases}
$$

Note that the formulae above may differ from some of the literature by either a sign or the replacement of left multiplication by right multiplication.

Let $w \in W_{\text {aff }}$ and consider $\iota_{w}: \mathrm{pt} \rightarrow \mathcal{G} / \mathcal{P}$ given by pt $\mapsto w \mathcal{P}$, the inclusion of a $T$-fixed point. Then the localization maps $\psi_{w}=\iota_{w}^{*}: H^{T}(\mathcal{G} / p) \rightarrow H^{T}(\mathrm{pt})=S$ form a basis for $H_{T}(\mathcal{G} / \mathcal{P})$ over $\operatorname{Frac}(S)$. Note that we have $\psi_{w}=\psi_{w^{\prime}}$ if $w \in w^{\prime} W_{P}$. The change of basis matrix between $\left\{\psi_{w}\right\}$ and $\left\{\sigma_{(w)}^{\mathcal{P}}\right\}$ is given by the $D$-matrix of [14, (4.21)]. The action of the nilHecke ring on the basis $\left\{\psi_{w}\right\}$ is given by

$$
v \cdot \psi_{w}=\psi_{v w} .
$$

Remark 3.3. In [28, the nilHecke ring $\mathbb{A}_{\text {aff }}$ is identified with $H_{T}(\mathcal{G} / \mathcal{B})$ via the map

$$
a \in \mathbb{A}_{\text {aff }} \longmapsto \psi_{\text {id }} \circ a \in \operatorname{Hom}_{S}\left(H^{T}(\mathcal{G} / \mathcal{B}), S\right) .
$$

One can give $H_{T}(\mathcal{G} / \mathcal{B})$ a product and coproduct which agrees with that of $\mathbb{A}_{\text {aff }}$ (see Section 3.3).

3.3. The coproduct on $\mathbb{A}_{\text {aff }}$. Define the coproduct map $\Delta: \mathbb{A}_{\text {aff }} \rightarrow \mathbb{A}_{\text {aff }} \otimes_{S} \mathbb{A}_{\text {aff }}$ by

$$
\begin{aligned}
\Delta(s) & =1 \otimes s=s \otimes 1
\end{aligned} \quad \text { for } s \in S,
$$

This is a well-defined map, which in addition is cocommutative. One can deduce from these relations that $\Delta(w)=w \otimes w$. (In the original work of [14, this last relation was used to define $\Delta$, but we shall follow the setup of [28].)

One should be careful since the tensor product $\mathbb{A}_{\mathrm{aff}} \otimes_{S} \mathbb{A}_{\mathrm{aff}}$ is not a ring in the obvious way. For example,

$$
\left(A_{i} \otimes 1\right) .\left(1 \otimes \alpha_{i}\right) \neq\left(A_{i} \otimes 1\right) \cdot\left(\alpha_{i} \otimes 1\right) .
$$

However, it is shown in 28 that the above formulae still give an action of $\mathbb{A}_{a f f}$ on $\mathbb{A}_{\text {aff }} \otimes_{S} \mathbb{A}_{\text {aff }}$. That is, $\Delta(a)=a \cdot(1 \otimes 1)$ for any $a \in \mathbb{A}$. In particular, if $M$ and $N$ are $\mathbb{A}_{\text {aff }}$ modules, then so is $M \otimes_{S} N$.

The following theorem of Kostant and Kumar in the non-equivariant case and Arabia in the equivariant case relates the coproduct on $\mathbb{A}_{\text {aff }}$ with the equivariant cohomology $H^{T}(\mathcal{G} / \mathcal{B})$.

Theorem 3.4 ([14, 1]). Let

$$
\Delta\left(A_{w}\right)=\sum_{u, v \in W_{\mathrm{aff}}} a_{w}^{u, v} A_{u} \otimes A_{v} .
$$

Then $a_{w}^{u, v}$ are the (Schubert) structure constants of $H^{T}(\mathcal{G} / \mathcal{B})$, so that

$$
\sigma_{\mathcal{B}}^{(u)} \cdot \sigma_{\mathcal{B}}^{(v)}=\sum_{w \in W_{\mathrm{aff}}} a_{w}^{u, v} \sigma_{B}^{(w)}
$$

where the $\sigma_{\mathcal{B}}^{(w)}$ denote the cohomology Schubert classes of $\mathcal{G} / \mathcal{B}$. 
Note that since $H^{T}(\mathcal{G} / \mathcal{P})$ imbeds into $H^{T}(\mathcal{G} / \mathcal{B})$, the structure constants for $\mathcal{G} / \mathcal{B}$ include the structure constants for $\mathcal{G} / \mathcal{P}$. Theorem 3.4 is in fact valid for all symmetrizable Kac-Moody groups.

\section{Equivariant homology of $\mathcal{G} r$ and Peterson's $j$-Homomorphism}

Peterson 28 has constructed an isomorphism, called $j$, between the equivariant homology $H_{T}(\mathcal{G} r)$ of the affine Grassmannian and the centralizer $Z_{\mathbb{A}_{\text {aff }}}(S)$ of $S$ in $\mathbb{A}_{\text {aff. }}$. We shall call the subalgebra $Z_{\mathbb{A}_{\text {aff }}}(S)$ the Peterson subalgebra. Since Peterson's work 28 has yet to be published, we include here a simplified construction of this isomorphism. Our definition of the isomorphism is more axiomatic than Peterson's, at the expense of being less natural; see Remark 4.6.

For this section we will think of $\mathcal{G} r$ both as the based loops $\Omega K$ and as $\mathcal{G} / \mathcal{P}$ for a maximal parabolic $\mathcal{P}$, but we will use the former notation. Note that $W_{P}=W$ in this case. Recall that $H_{T}(\Omega K)$ is the $S$-submodule of $\operatorname{Hom}_{S}\left(H^{T}(\Omega K), S\right)$ spanned by the classes $\sigma_{(w)}$. Over $\operatorname{Frac}(S), H_{T}(\Omega K)$ is also spanned by the classes $\left\{\psi_{t} \mid t=\right.$ $\left.t_{\lambda} \in Q^{\vee} \subset W_{\text {aff }}\right\}$.

Since $\Omega K$ is a group and the multiplication $\Omega K \times \Omega K \rightarrow \Omega K$ is $T$-equivariant, $H_{T}(\Omega K)$ and $H^{T}(\Omega K)$ obtain the structures of dual Hopf algebras. The Hopf structure maps of $H_{T}(\Omega K)$ can be calculated directly in terms of the $\psi_{t}$ as follows ( $\varepsilon$ denotes the counit and $c$ denotes the antipode):

$$
\begin{aligned}
& \psi_{\text {id }}=1, \quad \varepsilon\left(\psi_{t}\right)=1, \quad c\left(\psi_{t}\right)=\psi_{t^{-1}}, \\
& \Delta \psi_{t}=\psi_{t} \otimes \psi_{t}, \quad \psi_{t} \psi_{t^{\prime}}=\psi_{t t^{\prime}} .
\end{aligned}
$$

The scalars $s \in S \subset H_{T}(\Omega K)$ are central and $c(s)=s$. In particular, $H_{T}(\Omega K)$ is a commutative and co-commutative Hopf algebra; see also 3 for a comparison.

Lemma 4.1. Let $t_{\lambda} \in \mathbb{A}_{\mathrm{aff}}$ be the image of a translation $t_{\lambda} \in W_{\mathrm{aff}}$ in the nilHecke ring. Then $t_{\lambda} \in Z_{\mathbb{A}_{\text {aff }}}(S)$.

Proof. The translations $t_{\lambda}$ act on $Q_{\mathrm{aff}}=\bigoplus_{i \in I \cup\{0\}} \alpha_{i}$ via $t_{\lambda} \cdot(\alpha+n \delta)=\alpha+n \delta-$ $\langle\alpha, \lambda\rangle \delta$, where $\alpha \in R$ is a root of the finite root system. Since the image of $\delta$ in $S$ is 0 , we see that $t_{\lambda}$ lies in $Z_{\mathbb{A}_{\mathrm{aff}}}(S)$.

Definition 4.2. Define a map $j: H_{T}(\Omega K) \rightarrow \mathbb{A}_{\text {aff }}$ by

$$
\psi_{t} \longmapsto t
$$

for all $t \in Q^{\vee}$ and extending by linearity (over $S$ ).

From the definition, the image of $j$ lies in $\operatorname{Frac}(S) \otimes_{S} \mathbb{A}_{\text {aff }}$ but it turns out that the image of $j$ lies in $\left(\operatorname{Frac}(S) \otimes \operatorname{span}_{S}\left\{t \mid t \in Q^{\vee}\right\}\right) \cap \mathbb{A}_{\text {aff }}$. This will be clear after Lemma 4.3

Thus $j\left(H_{T}(\Omega K)\right)$ is the subalgebra of $\mathbb{A}_{\mathrm{aff}}$ generated by the elements $t \in Q^{\vee}$ and $s \in S$. Note that by Lemma 4.1] $t \in Z_{\mathbb{A}_{\text {aff }}}(S)$ so that the image of $j$ lies in $Z_{\mathbb{A}_{\mathrm{aff}}}(S)$ and in particular $j\left(H_{T}(\Omega K)\right)$ is an algebra over $S$.

Lemma 4.3. The map $j$ is a homomorphism of rings. It is compatible with the $\mathbb{A}_{\text {aff }}$ action on $H_{T}(\Omega K)$ as follows:

$$
\sigma \sigma^{\prime}=j(\sigma) \cdot \sigma^{\prime},
$$

for $\sigma, \sigma^{\prime} \in H_{T}(\Omega K)$. 
Proof. Since both $H_{T}(\Omega K)$ and $j\left(H_{T}(\Omega K)\right)$ are algebras over $S$ (that is, $S$ is central), we need only compute on the basis $\psi_{t}$. The first statement follows from $\psi_{t} \psi_{t^{\prime}}=\psi_{t t^{\prime}}$. The second statement follows from formula (3.3).

Every $A_{w}$ for $w \in W_{\text {aff }}$ acts non-trivially on $H_{T}(\Omega K)$. By writing $j(\sigma)=$ $\sum_{w \in A_{w}} a_{\sigma}^{w} A_{w}$ and using (3.1), Lemma 4.3 shows that the coefficients $a_{\sigma}^{w}$ lie in $S$, not $\operatorname{Frac}(S)$.

Theorem $4.4([28])$. There is an isomorphism $j: H_{T}(\Omega K) \rightarrow Z_{\mathbb{A}_{\mathrm{aff}}}(S)$ such that

$$
j\left(\sigma_{(x)}\right)=A_{x} \bmod I
$$

where $x \in W^{0}$ and

$$
I=\sum_{w \in W ; w \neq \mathrm{id}} \mathbb{A}_{\mathrm{aff}} \cdot A_{w}
$$

Proof. We have already shown that $j\left(H_{T}(\Omega K)\right) \subset Z_{\mathbb{A}_{\mathrm{aff}}}(S)$. By Lemma 4.3, we have $j\left(\sigma_{(x)}\right) \cdot \sigma_{(\mathrm{id})}=\sigma_{(x)}$ for $x \in W^{0}$. Using (3.2), we see that $j\left(\sigma_{(x)}\right)=A_{x}+a$ where $a$ lies in the annihilator of $\sigma_{(\mathrm{id})} \in H_{T}(\Omega K)$. This annihilator is easily seen to be equal to the left ideal $I$ defined above.

To show that $j$ is an isomorphism, write $a \in Z_{\mathbb{A}_{\text {aff }}}(S)$ as

$$
a=\sum_{w \in W_{\mathrm{aff}}} a_{w} w
$$

Now we use the commutation formula $w s=(w \cdot s) w$ in $\mathbb{A}_{\text {aff }}$ (see [14). Since $W$ acts faithfully on $S$, if $w \notin Q^{\vee}$, we must have $a_{w}=0$, and so $a \in j\left(H_{T}(\Omega K)\right)$.

The isomorphism of Theorem 4.4 can be made into a Hopf isomorphism by giving $Z_{\mathbb{A}_{\mathrm{aff}}}(S)$ the restriction of the coproduct $\Delta$ on $\mathbb{A}$ to $Z_{\mathbb{A}_{\mathrm{aff}}}(S)$. Since we have $\Delta(t)=t \otimes t \in Z_{\mathbb{A}_{\mathrm{aff}}}(S) \otimes_{S} Z_{\mathbb{A}_{\mathrm{aff}}}(S)$, this agrees with the coproduct of $H_{T}(\Omega K)$. We define the antipode by $c(t)=t^{-1}$ for $t \in Q^{\vee} \subset Z_{\mathbb{A}_{\mathrm{aff}}}(S)$ (see (4.1)). It is induced by $\alpha_{i} \mapsto-\alpha_{\omega(i)}$ for $i \in I\left(\alpha_{0} \mapsto-\alpha_{0}\right)$ and $A_{i} \mapsto-A_{\omega(i)}$ where $\omega$ is the diagram automorphism obtained by conjugation by the longest element of $W$.

The elements $j\left(\sigma_{\left(t_{\lambda}\right)}\right)$ for antidominant coweights $\lambda$ can be calculated explicitly.

Proposition 4.5. Let $x \in W^{0}$ and $\lambda \in Q^{-}$. Then we have

$$
\sigma_{(x)} \sigma_{\left(t_{\lambda}\right)}=\sigma_{\left(x t_{\lambda}\right)}
$$

in $H_{T}(\Omega K)$. Explicitly one has

$$
j\left(\sigma_{\left(t_{\lambda}\right)}\right)=\sum_{w \in W / W_{\lambda}} A_{t_{w \cdot \lambda}}
$$

where $W_{\lambda} \subset W$ is the stabilizer of $\lambda$.

Proof. Write $t:=t_{\lambda}$. First we note that $A_{i} \cdot \sigma_{(t)}=0$ for $i \neq 0$. This follows from (3.2), since using (2.1) one can check that for each $i \neq 0$ we have either $\ell\left(r_{i} t\right)<\ell(t)$ or $r_{i} t \notin W^{0}$. Now we compute, using Theorem 4.4 .

$$
\begin{array}{rlr}
\sigma_{(x)} \sigma_{(t)} & =j\left(\sigma_{(x)}\right) \cdot \sigma_{(t)} & \\
& =\left(A_{x}+a\right) \cdot \sigma_{(t)} & \text { where } a \in I, \\
& =A_{x} \cdot \sigma_{(t)} & \text { since } a \cdot \sigma_{(t)}=0, \\
& =\sigma_{(x t)} . &
\end{array}
$$


For the last statement, we write, using the fact that $H_{T}(\Omega K)$ is commutative, $\sigma_{(x t)}=j\left(\sigma_{(t)}\right) \cdot \sigma_{(x)}$. Now let $x=w t^{\prime}$ where $w \in W$ and $t^{\prime} \in Q^{\vee}$. Then

$$
x t=w t^{\prime} t_{\lambda}=w t_{\lambda} t^{\prime}=t_{w \cdot \lambda} w t^{\prime}
$$

so that $A_{t_{w \cdot \lambda}}$ must occur in the expansion of $j\left(\sigma_{(t)}\right)$. To obtain (4.4), by Theorem 4.4 it suffices to show that the element $a=\sum_{w \in W / W_{\lambda}} A_{t_{w \cdot \lambda}}$ lies in $Z_{\mathbb{A}_{\mathrm{aff}}}(S)$, since it is of the form $\left(A_{t} \bmod I\right)$.

Since the translations $t_{\nu}$ for any $\nu \in Q^{\vee}$ act as the identity on $S$, it suffices to show that all the terms of the second form in Lemma 3.1 occurring in $a \mu$ cancel, where $\mu \in h_{\mathbb{Z}}^{*} \subset S$. The real roots of $W_{\text {aff }}$ are of the form $\alpha+n \delta$ (with corresponding reflection $r_{\alpha} t_{n \alpha^{\vee}}$ ) where $\alpha \in R$ is a root of the finite root system and $n \in \mathbb{Z}$. The positive real roots are of the form $\alpha+n \delta$ where either $n>0$ or $n=0$ and $\alpha \in R^{+}$.

Suppose then that $t_{\nu} r_{\alpha} t_{n \alpha^{\vee}} \lessdot t_{\nu}$ and that $\alpha+n \delta$ is a positive root. By [13, Theorem 5.4] we have $t_{\nu} \cdot(\alpha+n \delta)=\alpha+(n-\langle\alpha, \nu\rangle) \delta<0$ which implies that $-\alpha+(\langle\alpha, \nu\rangle-n) \delta$ is a positive root. Since $\ell\left(t_{\nu}\right)=\ell\left(t_{r_{\alpha} \cdot \nu}\right)$ and

$$
t_{\nu} r_{\alpha} t_{n \alpha^{\vee}}=t_{r_{\alpha} \cdot \nu+\langle\alpha, \nu\rangle \alpha^{\vee}} r_{\alpha} t_{n \alpha^{\vee}}=t_{r_{\alpha} \cdot \nu} r_{\alpha} t_{(n-\langle\alpha, \nu\rangle) \alpha^{\vee}}
$$

we conclude that $A_{t_{\nu} r_{\alpha} t_{n \alpha} \vee}$ occurs in the expansion (via Lemma 3.1) of $A_{t_{\nu}} \mu$ and $A_{t_{r_{\alpha \cdot \nu}}} \mu$ with coefficients $\left\langle\mu, \alpha^{\vee}\right\rangle$ and $\left\langle\mu,-\alpha^{\vee}\right\rangle$, respectively (where we have used $\left.(\alpha+n \delta)^{\vee}=\alpha^{\vee}\right)$. Letting $\nu=w \cdot \lambda$, we see that these contributions cancel out in $a \mu$.

Remark 4.6. In [28, the map $j$ is obtained in the context of "compact characteristic operators" by considering the action

$$
\phi: \Omega K \times L K / T \longrightarrow L K / T
$$

of $\Omega K$ on $\mathcal{G} / \mathcal{B} \simeq L K / T$ where $L K$ is the space of all continuous loops into $K$. This action is obtained via the inclusion $\Omega K \hookrightarrow L K$ and is $T$-equivariant. Each $\sigma \in H_{T}(\Omega K)$ induces a composition

$$
H^{T}(L K / T) \longrightarrow H^{T}(\Omega K) \otimes_{S} H^{T}(L K / T) \longrightarrow S \otimes_{S} H^{T}(L K / T)
$$

where the first map is $\phi^{*}$ and the second map is $\sigma \otimes \mathrm{id}$. This defines a $H_{T}(\Omega K)$ module structure on $H^{T}(L K / T)$. If $\sigma \in H_{T}(\Omega K)$, then we obtain an operator $a_{\sigma}$ on $H^{T}(L K / T)$. This operator $a_{\sigma}$ acts on $H^{T}(L K / T)$ in the same way as $j(\sigma) \in \mathbb{A}_{\text {aff }}$. The expansion of $j(\sigma)$ in the $\left\{A_{w} \mid w \in W_{\text {aff }}\right\}$ basis can be obtained by calculating the action of $a_{\sigma}$ on the Schubert basis $\left\{\sigma^{(w)}\right\}$, using (3.1).

Note that since $\Omega K \hookrightarrow L K / T$ takes a fixed point $t_{\lambda} \in \Omega K$ to the fixed point $t_{\lambda} T$, it is immediate that this definition agrees with Definition 4.2 .

\section{Homology of affine Grassmannian and Fomin-Stanley subalgebra}

5.1. An identity for finite Weyl groups. In this subsection we let $W$ be a finite Weyl group and $H^{*}(K / T)$ the cohomology of the corresponding flag variety. Also let $w^{\circ}$ denote the longest element of $W$.

Proposition 5.1. Suppose that for some coefficients $\left\{b_{u} \in \mathbb{Z}\right\}_{u \in W}$ the following identity holds in $\mathbb{Z}[W]$ for all integral weights $\lambda \in h_{\mathbb{Z}}^{*}$ :

$$
\sum_{u \in W ; l(u)>0} b_{u} \sum_{u r_{\alpha} \lessdot u}\left\langle\lambda, \alpha^{\vee}\right\rangle u r_{\alpha}=0 .
$$

Then $b_{u}=0$ for all $u$. 
Proof. First apply the transformation $u \mapsto w^{\circ} u$ to the identity of the proposition. Then reindexing the $b_{u}$, we obtain

$$
\sum_{u \in W ; u \neq w^{\circ}} b_{u} \sum_{u r_{\alpha} \gg u}\left\langle\lambda, \alpha^{\vee}\right\rangle u r_{\alpha}=0
$$

for all $\lambda$.

Let $\zeta^{u} \in H^{*}(K / T)$ denote the cohomology Schubert classes of the finite flag variety. By the Chevalley-Monk formula [2] we have

$$
[\lambda] \cdot \zeta^{u}=\sum_{u r_{\alpha} \gg u}\left\langle\lambda, \alpha^{\vee}\right\rangle \zeta^{u r_{\alpha}}
$$

where $[\lambda] \in H^{*}(K / T)$ denotes the image of $\lambda \in h_{\mathbb{Z}}^{*}$ under the characteristic homomorphism $S\left(h_{\mathbb{Z}}^{*}\right) \rightarrow H^{*}(K / T)$. For example, if $\lambda=\omega_{i}$ is a fundamental weight, then $\left[\omega_{i}\right]=\zeta^{s_{i}}$. It is well known that $\zeta^{s_{1}}, \zeta^{s_{2}}, \ldots, \zeta^{s_{n-1}}$ generate the positive degree part of $H^{*}(K / T)$ or alternatively that the characteristic homomorphism is surjective.

Suppose that $[\lambda] \cdot \zeta=0$ for some $\zeta \in H^{*}(K / T)$ and all $\lambda \in h_{\mathbb{Z}}^{*}$. Then $\zeta^{u} \cdot \zeta=0$ for all $u \neq$ id. If $\ell(v)+\ell(u)=\ell\left(w_{\circ}\right)$, we have $\zeta^{v} \cdot \zeta^{u}=\delta_{v, w_{\circ} u} \zeta^{w_{\circ}}$. Thus we find that $\zeta$ must be a multiple of the class $\zeta^{w_{\circ}}$. Letting $\zeta=\sum_{u} b_{u} \zeta^{u}$ and applying the Chevalley-Monk formula, we obtain the proposition.

It is likely that Proposition 5.1 can also be phrased in terms of vanishing of Weyl group cohomology.

5.2. Affine Fomin-Stanley subalgebra. Let $\mathbb{A}_{0} \subset \mathbb{A}_{\text {aff }}$ denote the subring over $\mathbb{Z}$ of $\mathbb{A}_{\text {aff }}$ generated by the $A_{i}$ only, which we call the affine nilCoxeter algebra. There is a specialization map $\phi_{0}: \mathbb{A}_{\text {aff }} \rightarrow \mathbb{A}_{0}$ given by

$$
\phi_{0}: \sum_{w} a_{w} A_{w} \longmapsto \sum_{w} \phi_{0}\left(a_{w}\right) A_{w}
$$

where $\phi_{0}$ evaluates a polynomial $s \in S$ at 0 . Note that $\phi_{0}$ also sends $\mathbb{A}_{\text {aff }} \otimes_{S}$ $\mathbb{A}_{\text {aff }}$ to $\mathbb{A}_{0} \otimes_{\mathbb{Z}} \mathbb{A}_{0}$ by evaluating the coefficients at 0 when writing in the basis $\left\{A_{w} \otimes A_{v}\right\}_{w, v \in W_{\text {aff }}}$.

Define the affine Fomin Stanley subalgebra as the subspace $\mathbb{B}^{\prime} \subset \mathbb{A}_{0}$ satisfying

$$
\mathbb{B}^{\prime}=\left\{a \in \mathbb{A}_{0} \mid \phi_{0}(a s)=\phi_{0}(s) a \text { for all } s \in S\right\} .
$$

The name is justified by the following computation.

Lemma 5.2. The space $\mathbb{B}^{\prime}$ defined above is a subalgebra.

Proof. Let $a, b \in \mathbb{B}^{\prime}$ and $s \in S$. Then $b s=\phi_{0}(s) b+\sum_{w \in W_{\mathrm{aff}}} c_{w} A_{w}$ where $c_{w} \in S$ and $\phi_{0}\left(c_{w}\right)=0$. Thus

$$
\phi_{0}((a b) s)=a \phi_{0}(s) b+\sum_{w \in W_{\mathrm{aff}}} \phi_{0}\left(c_{w}\right) a A_{w}=\phi_{0}(s)(a b),
$$

and hence $a b \in \mathbb{B}^{\prime}$.

A combinatorial definition of the same algebra, in type $A$, will be given in Section 6.2 .

Proposition 5.3. Let $b \neq 0 \in \mathbb{B}^{\prime}$ and write $b=\sum_{w \in W_{\mathrm{aff}}} b_{w} A_{w}$ with $b_{w} \in \mathbb{Z}$. Then $b_{w} \neq 0$ for some $w \in W^{0}$. 
Proof. Let $D=\left\{w \in W_{\text {aff }} \mid b_{w} \neq 0\right\}$. For each $w \in W_{\text {aff we may uniquely write }}$ $w=x_{w} y_{w}$ where $x_{w} \in W^{0}$ and $y_{w} \in W$. Let $d=\left\{\min \left(\ell\left(y_{w}\right)\right) \mid w \in D\right\}$. We write $\ell_{0}(w):=\ell\left(y_{w}\right)$.

Suppose $d \neq 0$ and let $w \in D$ minimize $\ell_{0}(w)$. Let $\lambda \in S$ be of degree 1 . Then by Lemma 3.1. $\phi_{0}\left(A_{w} \lambda\right)=\sum_{w r_{\alpha} \lessdot w}\left\langle\lambda, \alpha^{\vee}\right\rangle A_{w r_{\alpha}}$. We know that $w>v$ if and only if a reduced decomposition of $v$ is obtained from a reduced decomposition of $w$ by removing a simple generator. Since $w=x_{w} y_{w}$, each such $v$ satisfies $\ell_{0}(v) \geq \ell_{0}(w)-1$. Let $D_{w}=\left\{v \lessdot w \mid \ell_{0}(v)=\ell_{0}(w)-1\right\}$. Then $v \in D_{w}$ if and only if $v=x_{v} y_{v}$ where $x_{v}=x_{w}$ and $y_{v} \lessdot y_{w}$. In this case $v=w r_{\alpha}$, where $\alpha \in R$ is a root of the finite root system.

Now write $\phi_{0}(b \lambda)=\sum_{v} b_{v}^{\prime} A_{v}$ and focus only on the coefficients of $b_{v}^{\prime}$ satisfying $\ell_{0}(v)=d-1$ and $v=x y_{v}$ for some fixed $x \in W^{0}$. If $b \in \mathbb{B}^{\prime}$, then $b_{v}^{\prime}=0$. Thus in particular, for every $\lambda \in S$ of degree 1 , we have

$$
\sum_{u \in W \mid \ell(u)=d} b_{x u} \sum_{u r_{\alpha} \lessdot u}\left\langle\lambda, \alpha^{\vee}\right\rangle A_{x} A_{u r_{\alpha}}=0 .
$$

Now the set $\left\{A_{x} A_{u} \mid u \in W\right\}$ is independent over $S$. So by replacing $A_{x} A_{u}$ by $u \in \mathbb{Z}[W]$, we see that this is impossible by Proposition 5.1. Thus we conclude that we must have $d=0$.

The following proposition says that evaluation at 0 maps the Peterson subalgebra onto the affine Fomin-Stanley subalgebra.

Proposition 5.4. We have $\phi_{0}\left(Z_{\mathbb{A}_{\mathrm{aff}}}(S)\right)=\mathbb{B}^{\prime}$. More precisely, the set of elements $\left\{\phi_{0}\left(j\left(\sigma_{(u)}\right)\right) \mid u \in W^{0}\right\}$ forms a basis of $\mathbb{B}^{\prime}$ over $\mathbb{Z}$. Furthermore, the element $\phi_{0}\left(j\left(\sigma_{(u)}\right)\right)$ is the unique element in $\mathbb{B}^{\prime}$ with unique Grassmannian term $A_{u}$.

Proof. If $a \in Z_{\mathbb{A}_{\text {aff }}}(S)$, then $\phi_{0}\left(\phi_{0}(a) s\right)=\phi_{0}(a s)=\phi_{0}(s a)=\phi_{0}(s) \phi_{0}(a)$, so that $\phi_{0}\left(Z_{\mathbb{A}_{\mathrm{aff}}}(S)\right) \subset \mathbb{B}^{\prime}$. Now let $b \in \mathbb{B}^{\prime}$. By Proposition 5.3 it contains a Grassmannian term $A_{u}$ with non-zero coefficient $b_{u}$. By Theorem 4.4, $b-b_{u} \phi_{0}\left(j\left(\sigma_{(u)}\right)\right)$ has strictly fewer Grassmannian terms and also lies in $\mathbb{B}^{\prime}$. Repeating, we see that one can write $b$ uniquely as a $\mathbb{Z}$-linear combination of the elements $\phi_{0}\left(j\left(\sigma_{(u)}\right)\right)$. The last statement follows from Theorem 4.4 .

By Theorem 4.4, we have $\Delta(z) \in Z_{\mathbb{A}_{\mathrm{aff}}}(S)$ for $z \in Z_{\mathbb{A}_{\mathrm{aff}}}(S)$ where $\Delta$ is the coproduct of $\mathbb{A}_{\text {aff }}$ introduced in Section 3.3. Applying the evaluation $\phi_{0}$ (and using Theorem (3.4), we see that $\mathbb{B}^{\prime}$ is closed under the operation $\Delta_{\mathbb{B}^{\prime}}=\phi_{0} \circ \Delta$. Thus $\mathbb{B}^{\prime}$ attains the structure of a Hopf algebra over $\mathbb{Z}$.

Since we have $H^{*}(\mathcal{G} r)=H^{T}(\mathcal{G} r) \otimes_{S} \mathbb{Z}$, where $S$ acts on $\mathbb{Z}$ by evaluation at 0 , the product and coproduct structure constants of $H^{*}(\mathcal{G} r)$ (respectively $H_{*}(\mathcal{G} r)$ ) are obtained from those of $H^{T}(\mathcal{G} r)$ (respectively $H_{T}(\mathcal{G} r)$ ) by evaluating at 0 . We thus obtain the following theorem.

Theorem 5.5. The affine Fomin-Stanley subalgebra $\mathbb{B}^{\prime}$ is a model for the homology $H_{*}(\mathcal{G} r)$. More precisely, the map $H_{*}(\mathcal{G} r) \rightarrow \mathbb{B}^{\prime}$ given by $\sigma_{u} \mapsto \phi_{0}\left(j\left(\sigma_{(u)}\right)\right)$ is an isomorphism of Hopf algebras.

As a consequence of the commutativity of $H_{*}(\mathcal{G} r)$ we have the following result.

Corollary 5.6. The affine Fomin-Stanley subalgebra $\mathbb{B}^{\prime}$ is a commutative algebra.

It does not seem easy to establish Corollary 5.6 directly from its definition. In the forthcoming sections, our aim will be to explicitly describe the elements $\phi_{0}\left(j\left(\sigma_{(u)}\right)\right)$ 
in the type $A_{n-1}$ case, that is, with $K=S U(n)$. This explicit description, combined with the combinatorial work in [17, gives a direct proof of Corollary [5.6 in type $A_{n-1}$.

Remark 5.7. As will be clear shortly, it is reasonable to call the homology Schubert basis elements $\phi_{0}\left(j\left(\sigma_{(u)}\right)\right)$ "non-commutative $k$-Schur functions" for the group $G$. The element $\phi_{0}\left(j\left(\sigma_{(u)}\right)\right)$ is characterized by being an element of $\mathbb{B}^{\prime}$ and having a unique Grassmannian term $A_{u}$. It would be interesting to describe $\phi_{0}\left(j\left(\sigma_{(u)}\right)\right)$ explicitly for arbitrary Lie types.

\section{6. $k$-SCHUR FUNCTIONS}

For the remainder of the paper, unless specified otherwise, we will restrict ourselves to type $A_{n-1}$. We have $G=S L(n, \mathbb{C}), K=S U(n), \mathcal{G}=S L(n, \mathbf{F})$. Thus $W=S_{n}$ is the symmetric group, $W_{\text {aff }}$ is the affine symmetric group and the affine simple roots are given by $\left\{\alpha_{i} \mid i \in \mathbb{Z} / n \mathbb{Z}\right\}$.

6.1. Symmetric functions. We refer to 27] for details concerning the material of this section. Let $\Lambda=\Lambda_{\mathbb{Z}}$ denote the ring of symmetric functions over $\mathbb{Z}$ in infinitely many variables $x_{1}, x_{2}, \ldots$. We write $h_{i}(x)$ for the homogeneous symmetric functions and for a partition $\lambda=\left(\lambda_{1} \geq \lambda_{2} \geq \cdots\right)$, we write $h_{\lambda}(x)=h_{\lambda_{1}(x)} h_{\lambda_{2}(x)} \cdots$. The elements $h_{1}(x), h_{2}(x), \ldots \in \Lambda$ form a set of algebraically independent generators of $\Lambda$. Similarly, we let $e_{i}(x)$ denote the elementary symmetric functions. The algebra involution $\omega$ of $\Lambda$ is defined by setting $\omega\left(h_{i}(x)\right)=e_{i}(x)$.

We let $\left\{m_{\lambda}(x) \in \Lambda \mid \lambda\right.$ a partition $\}$ denote the monomial symmetric functions. They form a basis of the ring of symmetric functions over the integers. Also let $p_{k}(x)$ denote the power sum symmetric functions. The power sum symmetric functions are algebraically independent generators of the ring of symmetric functions over $\mathbb{Q}$.

Let $\Lambda_{n} \subset \Lambda$ denote the subring of the symmetric functions generated by $h_{i}(x)$ for $i \in[0, n-1]$. Let $\Lambda^{n}$ denote the quotient algebra of $\Lambda$ given by $\Lambda^{n}=\Lambda /\left\langle m_{\lambda}(x)\right|$ $\left.\lambda_{1} \geq n\right\rangle$. Clearly the set $\left\{m_{\lambda}(x) \mid \lambda_{1}<n\right\}$ forms a basis of $\Lambda^{n}$. When giving an element $\bar{f} \in \Lambda^{n}$, we will usually just give a representative $f \in \Lambda$ without further comment.

The Hall inner product, denoted $\langle.,\rangle:. \Lambda \times \Lambda \rightarrow \mathbb{Z}$, is a symmetric non-degenerate pairing defined by $\left\langle h_{\lambda}(x), m_{\mu}(x)\right\rangle=\delta_{\lambda \mu}$. It induces a non-degenerate pairing $\langle.,$.$\rangle :$ $\Lambda_{n} \times \Lambda^{n} \rightarrow \mathbb{Z}$. The ring of symmetric functions $\Lambda$ can be given the structure of a (commutative and co-commutative) Hopf algebra with coproduct $\Delta_{\text {Sym }}: \Lambda(x) \rightarrow$ $\Lambda(x) \otimes \Lambda(y)$ given by $\Delta_{\text {Sym }}\left(h_{i}(x)\right)=\sum_{0<j<i} h_{j}(x) \otimes h_{i}(y)$, where $h_{0}=1$. The coproduct can alternatively be given by specifying $\Delta_{\mathrm{Sym}}\left(p_{i}(x)\right)=p_{i}(x) \otimes 1+1 \otimes$ $p_{i}(y)$. This Hopf algebra structure gives $\Lambda_{n}$ and $\Lambda^{n}$ the structures of dual Hopf algebras. The counit $\varepsilon$ is given by the constant term $\varepsilon(f(x))=f(0)$. The antipode $c$ is given by $c\left(h_{i}(x)\right)=(-1)^{i} e_{i}(x)$; thus if $f(x) \in \Lambda$ is homogeneous of degree $d$, then $c(f(x))=(-1)^{d} \omega(f(x))$.

The homology and cohomology rings (and their Hopf algebra structures) of $\Omega K=\Omega S U(n)$ (in fact for the based loops of all compact Lie groups) were earlier computed by Bott.

Theorem 6.1 (4, Proposition 8.1]). We have an isomorphism in homology

$$
H_{*}(\mathcal{G} / \mathcal{P})=\mathbb{Z}\left[\sigma_{1}, \sigma_{2}, \ldots, \sigma_{n-1}\right]
$$


where $\operatorname{dim} \sigma_{i}=2 i$ and the coproduct is given by $\Delta\left(\sigma_{i}\right)=\sum_{0 \leq j \leq i} \sigma_{j} \times \sigma_{i-j}$ where $\sigma_{0}=1$. In cohomology we have

$$
H^{*}(\mathcal{G} / \mathcal{P})=\mathcal{S} H^{*}\left(\mathbb{C P}^{n-1}\right)
$$

where $\mathcal{S}$ denotes an infinite symmetric power. The primitive subspace of $H^{*}(\mathcal{G} / \mathcal{P})$ is the space spanned by the power sums $p_{k}(u)=u_{1}^{k}+u_{2}^{k}+\cdots$ where the $u_{i}$ are the generators of the different copies of $H^{*}\left(\mathbb{C P}^{n-1}\right) \simeq \mathbb{Z}[u] / u^{n}$.

Thus the homology $H_{*}(\mathcal{G} / \mathcal{P})$ can be identified with the subring of symmetric functions $\Lambda_{n}$ via $\sigma_{i} \mapsto h_{i}$ while the cohomology $H^{*}(\mathcal{G} / \mathcal{P})$ can similarly be identified with the quotient $\Lambda^{n}$. The aim of this paper is thus to identify the Schubert classes $\sigma_{w} \in H_{*}(\mathcal{G} / \mathcal{P}) \simeq \Lambda_{n}$ and $\sigma^{w} \in H^{*}(\mathcal{G} / \mathcal{P}) \simeq \Lambda^{n}$ as explicit symmetric functions.

Note that because of the nature (one dimensional for each graded piece with degree from 0 to $n-1$ ) of the primitive subspace of $H^{*}(\mathcal{G} / \mathcal{P})$, there is essentially no choice in these Hopf isomorphisms. The only flexibility would be sending $u_{1}^{k}+$ $u_{2}^{k}+\cdots$ to the negative power sum $-p_{k}$ instead of $p_{k}$. This is ruled out if we want the Schubert classes $\sigma^{w}$ to be represented by symmetric functions with a positive monomial expansion.

6.2. Affine Schur functions and $k$-Schur functions. In this section we define dual bases $\left\{s_{\lambda}^{(k)}(x)\right\}$ of $\Lambda_{n}$ and $\left\{F_{\lambda}(x)\right\}$ of $\Lambda^{n}$ called, respectively, the $k$-Schur functions and the affine Schur functions or dual $k$-Schur functions. Historically, the $k$-Schur functions $\left\{s_{\lambda}^{(k)}(x)\right\}$ were introduced first in 21] and were further studied in 22,23 . Their introduction was motivated by a series of conjectures the most striking of which is their relationship with the Macdonald polynomials, which we briefly explain.

The original $k$-Schur functions $s_{\lambda}^{(k)}(x ; t)$ depended on an extra parameter $t$, and the $k$-Schur functions we use are the $t=1$ specializations: $s^{(k)}(x)=s_{\lambda}^{(k)}(x ; 1)$. Let $H_{\mu}(x ; q, t)$ be given by the plethystic substitution

$$
H_{\mu}(x ; q, t)=J_{\mu}(x /(1-q) ; q, t)
$$

where $J_{\mu}(x ; q, t)$ is the integral form of Macdonald polynomials [27]. Define $K_{\nu \mu}^{(k)}(q, t)$ $\in \mathbb{Z}[q, t]$ and $\pi_{\lambda \nu}^{(k)}(t) \in \mathbb{Z}[q, t]$ by

$$
H_{\mu}(x ; q, t)=\sum_{\nu} K_{\nu \mu}^{(k)}(q, t) s_{\nu}^{(k)}(x ; t) ; \quad s_{\nu}^{(k)}(x ; t)=\sum_{\lambda} \pi_{\lambda \nu}^{(k)}(t) s_{\lambda}(x) .
$$

Here $s_{\lambda}(x)$ denotes a Schur function. Then it is conjectured that $K_{\nu \mu}^{(k)}(q, t) \in$ $\mathbb{N}[q, t]$ and $\pi_{\lambda \nu}^{(k)}(t) \in \mathbb{N}[t]$ which would refine the (proven) "Macdonald positivity conjecture" that the Schur expansion of $H_{\mu}(x ; q, t)$ has coefficients in $\mathbb{N}[q, t]$; see [12].

There are a number of conjecturally equivalent definitions of $k$-Schur functions. We will use a reformulation of the definition from [23. which involves first defining the affine Stanley symmetric functions from [17].

Definition $6.2\left([17)\right.$. Let $a=a_{1} a_{2} \cdots a_{k}$ be a word with letters from $\mathbb{Z} / n \mathbb{Z}$ so that $a_{i} \neq a_{j}$ for $i \neq j$. Let $A=\left\{a_{1}, a_{2}, \ldots, a_{k}\right\} \subset \mathbb{Z} / n \mathbb{Z}$. The word $a$ is cyclically decreasing if for every $i$ such that $i, i+1 \in A$, the letter $i+1$ precedes $i$ in $a$. An affine permutation $w \in W_{\text {aff }}$ is cyclically decreasing if $w=s_{a_{1}} \cdots s_{a_{k}}$ for some cyclically decreasing sequence $a_{1} a_{2} \cdots a_{k}$. 
Define the elements $h_{i} \in \mathbb{A}_{0} \subset \mathbb{A}_{\text {aff }}: i \in[0, n-1]$ by the formula

$$
h_{i}=\sum_{w} A_{w}
$$

where the sum is over all cyclically decreasing permutations $w \in W_{\text {aff }}$ with length $\ell(w)=i$. If $I \subset \mathbb{Z} / n \mathbb{Z}$ and $w$ is the corresponding cyclically decreasing permutation, then we will write $A_{I}$ for $A_{w}$. This gives a one-to-one correspondence between cyclically decreasing permutations and subsets of $\mathbb{Z} / n \mathbb{Z}$. Here $h_{0}=A_{\text {id }}=1$. As an example, with $n=4$ and $i=2$ we have $h_{3}=A_{3} A_{2}+A_{3} A_{1}+A_{0} A_{3}+A_{2} A_{1}+$ $A_{2} A_{0}+A_{1} A_{0}$.

Let $\mathbb{B}$ denote the subalgebra of $\mathbb{A}_{0} \subset \mathbb{A}$ generated by the $h_{i}$ for $i \in[0, n-1]$, which we call the affine Fomin-Stanley subalgebra (we will show that $\mathbb{B}$ agrees with $\mathbb{B}^{\prime}$ defined in Section 5.2).

Theorem 6.3 ([17). The algebra $\mathbb{B}$ is commutative. It is isomorphic to the subalgebra $\Lambda_{n}$ of the symmetric functions generated by the homogeneous symmetric functions $h_{i}(x)$ for $i \in[0, n-1]$, under the map $\psi: h_{i}(x) \mapsto h_{i} \in \mathbb{B}$.

Let $\langle.,\rangle:. \mathbb{A}_{0} \times \mathbb{A}_{0} \rightarrow \mathbb{Z}$ denote the symmetric non-degenerate pairing defined by $\left\langle A_{w}, A_{v}\right\rangle=\delta_{w v}$.

Definition 6.4 ([17, Definition 3]). Let $w \in W_{\text {aff }}$. Define the affine Stanley symmetric functions $\tilde{F}_{w}(x) \in \Lambda$ by

$$
\tilde{F}_{w}(x)=\sum_{a=\left(a_{1}, a_{2}, \ldots, a_{t}\right)}\left\langle h_{a_{t}} h_{a_{t-1}} \cdots h_{a_{1}} \cdot 1, A_{w}\right\rangle x_{1}^{a_{1}} x_{2}^{a_{2}} \cdots x_{t}^{a_{t}},
$$

where the sum is over compositions of $\ell(w)$ satisfying $a_{i} \in[0, n-1]$.

This definition is similar to the definition of Stanley symmetric functions given by Fomin and Stanley [7. For example, let $n=3$ and $w=s_{1} s_{2} s_{1} s_{0}$. Then $\tilde{F}_{w}\left(x_{1}, x_{2}, x_{3}, x_{4}\right)=2 x_{1} x_{2} x_{3} x_{4}+\left(x_{1}^{2} x_{2} x_{3}+x_{1} x_{2}^{2} x_{3}+x_{1} x_{2} x_{3}^{2}+x_{1}^{2} x_{2} x_{4}+\cdots\right)$.

The image in $\Lambda^{n}$ of the set $\left\{\tilde{F}_{w}(x) \mid w \in W^{0}\right\}$ forms a basis of $\Lambda^{n}$ (see [17, 24]). We called these functions affine Schur functions in [17. They were earlier introduced in a different manner in 24, where they were called dual $k$-Schur functions.

Define the $k$-Schur functions $\left\{s_{w}^{(k)}(x) \mid w \in W^{0}\right\}$ as the dual basis of $\Lambda_{n}$ to the affine Schur functions under the Hall inner product, where $k=n-1$. There is a bijection $w \leftrightarrow \lambda(w)$ from affine Grassmannian permutations $\left\{w \in W^{0}\right\}$ to partitions $\left\{\lambda \mid \lambda_{1}<n\right\}$ obtained by taking the code of the permutation; see [17, 19. If we make the identifications $\tilde{F}_{w}(x)=\tilde{F}_{\lambda(w)}(x)$ and $s_{w}^{(k)}(x)=s_{\lambda(w)}^{(k)}(x)$ under this bijection, then this agrees with the usual indexing of $k$-Schur functions and affine Schur functions by partitions; see [17. In [19, Theorem 5.10], the monomial expansion of the $s_{w}^{(k)}(x)$ is given in terms of certain chains obtained from the strong order of the affine symmetric group. These chains can be made combinatorially explicit, using the language of cores, in a manner similar to the classical definition of Schur functions (see [19, Sections 10-11]).

6.3. Non-commutative $k$-Schur functions. The following definition was inspired by work of Fomin and Greene [6]. Recall the isomorphism $\psi: \Lambda_{n} \simeq \mathbb{B}$ of Theorem 6.3 
Definition 6.5. Let $w \in W^{0}$. The non-commutative $k$-Schur functions are given by

$$
s_{w}^{(k)}:=\psi\left(s_{w}^{(k)}(x)\right) \in \mathbb{B} .
$$

Example 6.6. Let $n=3=k+1$. The affine Grassmannian permutations with length less than or equal to 3 are id, $s_{0}, s_{1} s_{0}, s_{2} s_{0}, s_{2} s_{1} s_{0}$ and $s_{1} s_{2} s_{0}$. Here we have computed the corresponding non-commutative $k$-Schur functions, where we write $A_{i_{1} i_{2} \cdots i_{k}}$ for $A_{i_{1}} \cdots A_{i_{k}}=A_{s_{i_{1}} s_{i_{2}} \cdots s_{i_{k}}}$.

$$
\begin{aligned}
s_{\mathrm{id}}^{(k)} & =1, \\
s_{s_{0}}^{(k)} & =h_{1}=A_{0}+A_{1}+A_{2}, \\
s_{s_{1} s_{0}}^{(k)} & =h_{2}=A_{02}+A_{21}+A_{10}, \\
s_{s_{2} s_{0}}^{(k)} & =h_{1}^{2}-h_{2}=A_{20}+A_{12}+A_{01}, \\
s_{s_{2} s_{1} s_{0}}^{(k)} & =h_{2} h_{1}=h_{1} h_{2}=A_{021}+A_{010}+A_{102}+A_{121}+A_{202}+A_{210}, \\
s_{s_{1} s_{2} s_{0}}^{(k)} & =h_{1}^{3}-h_{2} h_{1}=A_{120}+A_{010}+A_{201}+A_{121}+A_{202}+A_{012} .
\end{aligned}
$$

Observe that all the coefficients in the above formulae are non-negative. Also note that $s_{w}^{(k)}$ contains only one term corresponding to a Grassmannian permutation and this term is $A_{w}$. These properties are explained in Proposition 6.7 and Corollary 8.5.

The main result we need concerning the non-commutative $k$-Schur functions is the following. Define the coefficients $b_{w, v} \in \mathbb{Z}$, where $w \in W^{0}$ and $v \in W_{\text {aff }}$, by

$$
s_{w}^{(k)}=\sum_{v \in W_{\text {aff }}} b_{w, v} A_{v} .
$$

Proposition 6.7 ([17, Proposition 42]). The coefficient $b_{w, v}$ is equal to the coefficient of $\tilde{F}_{w}$ in the affine Stanley function $\tilde{F}_{v}$, when written in the basis $\left\{\tilde{F}_{w}\right.$ : $\left.w \in W^{0}\right\}$ of affine Schur symmetric functions. In particular, $s_{w}^{(k)}$ has a unique Grassmannian term $A_{w}$.

In [17, Conjecture 38], we conjectured that the $b_{w, v}$ were positive and showed how they implied positivity conjectures related to the toric Schur functions of Postnikov [29] and $k$-Schur functions [21]. We shall return to these coefficients in Section 8

We now describe the Hopf structure on $\mathbb{B}$, acquired under the isomorphism $\psi$ : $\Lambda_{n} \simeq \mathbb{B}$. We let the unit be $h_{0}=A_{\text {id }}$ and we let the counit $\varepsilon(b)$ be the coefficient of $h_{0}$ in $b$ when written as a polynomial in the $h_{i}$.

The coproduct $\Delta_{\mathbb{B}}: \mathbb{B} \rightarrow \mathbb{B} \otimes_{\mathbb{Z}} \mathbb{B}$ is given by

$$
\Delta_{\mathbb{B}}\left(h_{i}\right)=\sum_{j \leq i} h_{j} \otimes h_{i-j}
$$

and by extending $\Delta_{\mathbb{B}}$ to a ring homomorphism.

Now define $\omega_{\mathbb{B}}: \mathbb{B} \rightarrow \mathbb{B}$ by $\omega_{\mathbb{B}}\left(h_{i}\right)=\psi\left(e_{i}(x)\right)$. Let ${ }^{-}: \mathbb{A}_{0} \rightarrow \mathbb{A}_{0}$ be the algebra involution of the nilCoxeter algebra induced by ${ }^{-}: A_{i} \mapsto A_{n-i}$, where $A_{0}=A_{n}$ is fixed by the involution. The following result is essentially Proposition 16 of [17.

Proposition 6.8. Let $b \in \mathbb{B}$. Then $\omega_{B}(b)=\bar{b}$.

For example, with $n=3$ as in Example 6.6, $\omega_{B}\left(s_{s_{1} s_{0}}^{(k)}\right)=s_{s_{2} s_{0}}^{(k)}$. We give $\mathbb{A}_{0}$ a grading (half of the topologically induced one) by letting $\operatorname{deg} A_{i}=1$. Thus 
for a homogeneous element $b \in \mathbb{B}$ with degree $d$ (as an element of $\mathbb{A}_{0}$ ) we define the antipode to be $c_{\mathbb{B}}(b)=(-1)^{d} \bar{b}$, agreeing with the antipode of the symmetric functions.

\section{Affine Grassmannian Schubert polynomials}

Our main result is the following.

Theorem 7.1. The map $\theta: H_{*}(\mathcal{G} / \mathcal{P}) \rightarrow \Lambda_{n}$ given by

$$
\theta: \sigma_{w} \longmapsto s_{w}^{(k)}(x)
$$

is an isomorphism of Hopf algebras. The map $\theta^{\prime}: H^{*}(\mathcal{G} / \mathcal{P}) \rightarrow \Lambda^{n}$ given by

$$
\theta^{\prime}: \sigma^{w} \longmapsto \tilde{F}_{w}(x)
$$

is an isomorphism of Hopf algebras.

In the homology case, this theorem was a conjecture of Mark Shimozono, who based it on computer calculations using the nilHecke ring and Theorem 3.4. The conjecture in the cohomology case was made precise by Jennifer Morse.

Theorem 7.1 will follow from two technical computations comparing $\mathbb{B}$ and $\mathbb{B}^{\prime}$ as Hopf algebras, which we now state.

Proposition 7.2. Let $b \in \mathbb{B}$ and $s \in S$. Then

$$
\phi_{0}(b s)=\phi_{0}(s) b \text {. }
$$

In other words, we have $\mathbb{B} \subset \mathbb{B}^{\prime}$.

Proposition 7.3. The two coproducts $\Delta$ and $\Delta_{\mathbb{B}}$ agree on $\mathbb{B}$ up to specialization at 0 :

$$
\left(\phi_{0} \circ \Delta\right)(b)=\Delta_{\mathbb{B}}(b),
$$

for $b \in \mathbb{B}$.

Theorem 7.4. The two algebras $\mathbb{B}$ and $\mathbb{B}^{\prime}$ are identical as subalgebras of $\mathbb{A}_{0}$. Furthermore the two Hopf structures agree and we have for each $w \in W^{0}$,

$$
\phi_{0}\left(j\left(\sigma_{(w)}\right)\right)=s_{w}^{(k)} \text {. }
$$

Proof. By Proposition 6.7 the element $s_{w}^{(k)} \in \mathbb{B}$ has a unique Grassmannian term $A_{w}$. By Proposition 7.2, it lies in $\mathbb{B}^{\prime}$, so by Proposition 5.4, we have $\phi_{0}\left(j\left(\sigma_{(w)}\right)\right)=$ $s_{w}^{(k)}$ and the elements $s_{w}^{(k)}$ span $\mathbb{B}^{\prime}$ so we conclude that $\mathbb{B}=\mathbb{B}^{\prime}$. For the Hopf algebra structure, Proposition 7.3 shows that the coproduct agrees. The agreement of the unit and counit is easy to see.

Finally, the antipode of $\mathbb{B}^{\prime}$ (described after Theorem 4.4 for any simple Lie type) is given by $t \mapsto t^{-1}$ which is induced by the map $A_{i} \mapsto-A_{\omega(i)}$ for each $i \in I$, where $\omega$ is the diagram automorphism of the finite Dynkin diagram and $\omega(0)=0$. In type $A_{n-1}$, this is given by $A_{i} \mapsto-A_{n-i}$. This agrees with the antipode $c_{\mathbb{B}}$ of $\mathbb{B}$ described in Section 6.3

Proof of Theorem 7.1. For homology the theorem follows immediately from Theorem 7.4 via the composition

$$
H_{*}(\mathcal{G} r) \longrightarrow \mathbb{B} \simeq \mathbb{B}^{\prime} \stackrel{\psi^{-1}}{\longrightarrow} \Lambda_{n},
$$

and the statement for cohomology follows by duality. 
Remark 7.5. Note that Proposition 7.3, together with Proposition 6.7 already implies that the coproduct of $k$-Schur functions agrees with the product in cohomology. Briefly, the fact that each non-commutative $k$-Schur function has a unique Grassmannian "leading term" allows one to obtain a coproduct expansion agreeing with Theorem 3.4 for Grassmannian permutations. This is the argument given in [18.

Remark 7.6. We find two symmetric function interpretations of Theorem 7.1 and Theorem 7.4 rather curious.

First, the Hopf algebra structure of the symmetric functions (which has been studied from many perspectives) now attains a topological origin. In the classical identification of the Schur functions as Schubert classes of the Grassmannian, only the product (not coproduct) of the symmetric functions is used. In particular it is interesting that the Hall inner product is now given an interpretation as the pairing between homology and cohomology.

Secondly, the commutativity of $\mathbb{B}=\mathbb{B}^{\prime}$ links the symmetry of affine Stanley symmetric functions with the commutativity of $H_{*}(\mathcal{G} r)$, which in turn is due to the fact that $\mathcal{G} r$ is a double loop space. Note that as shown in [17, the usual Stanley symmetric functions $F_{w}$ are special cases of affine Stanley symmetric functions, so we have now a "topological" explanation of the symmetry of Stanley symmetric functions.

7.1. Proof of Proposition 7.2; $\mathbb{B}^{\prime}$ contains $\mathbb{B}$. Suppose $a \in \mathbb{A}_{0}$ and $s, s^{\prime} \in S$. Then $\phi_{0}\left(a s s^{\prime}\right)=\phi_{0}\left(\phi_{0}(a s) s^{\prime}\right)$. Since $\phi_{0}$ is linear (over $\mathbb{Z}$ ) and the $\left\{\alpha_{j}\right\}_{j \in \mathbb{Z} / n \mathbb{Z}}$ generate the elements of $S$ with constant term 0 , to show that $h_{i} \in \mathbb{B}^{\prime}$ it suffices to show that $\phi_{0}\left(h_{i} \cdot \alpha_{j}\right)=0$ for each $i$. The result will then follow from the fact that the $\left\{h_{i}\right\}_{i=0}^{n-1}$ generate $\mathbb{B}$ and that $\mathbb{B}^{\prime}$ is an algebra.

Without loss of generality we will assume that $j=1$. Let $I \subset \mathbb{Z} / n \mathbb{Z}$ be of size $i$. We calculate $\phi_{0}\left(A_{I} \alpha_{1}\right)$ explicitly. In the following table we let $[2, r]$ be the largest interval of its form (possibly empty) contained in $I$ which contains 2 . It is possible that $[2, r]$ contains 0 but it cannot contain 1 (since then it will have size $n$ ). Also the subset $I^{\prime}$ never contains any of $0,1,2$. The sums over $a$ are always over $a \in[2, r]$. The (A),(B),(C) are for marking the terms only, for later use.

\begin{tabular}{|c|c|c|}
\hline & $I$ & $\phi_{0}\left(A_{I} \alpha_{1}\right)$ \\
\hline $0,1 \notin I$ & $I^{\prime} \cup[2, r]$ & $-\sum_{a} A_{I-\{a\}}(\mathrm{A})$ \\
$1 \in I$ and $0 \notin I$ & $I^{\prime} \cup[2, r] \cup\{1\}$ & $2 A_{I-\{1\}}(\mathrm{A})+\sum_{a} A_{I-\{a\}}(\mathrm{C})$ \\
$0 \in I$ and $1 \notin I$ & $I^{\prime} \cup[2, r] \cup\{0\}$ & $-A_{I-\{0\}}(\mathrm{A})-\sum_{a} A_{I-\{a\}}(\mathrm{B})$ \\
$0,1 \in I$ & $I^{\prime} \cup[2, r] \cup\{0,1\}$ & $-A_{I-\{0\}}(\mathrm{C})+A_{I-\{1\}}(\mathrm{B})$ \\
\hline
\end{tabular}

For example

$$
\begin{aligned}
A_{[2, r]} A_{1} A_{0} \alpha_{1} & \\
& =A_{[2, r]} A_{1}\left(\left(\alpha_{1}+\alpha_{0}\right) A_{0}-1\right) \\
& =-A_{[2, r]} A_{1}+A_{[2, r]}\left(-\alpha_{1} A_{1} A_{0}+2 A_{0}+\left(\alpha_{1}+\alpha_{0}\right) A_{1} A_{0}-A_{0}\right) \\
& =-A_{[2, r]-\{0\}}+A_{[2, r]-\{1\}}+\alpha_{0} A_{[2, r]} A_{1} A_{0} .
\end{aligned}
$$

The $A_{t}$ factors for $t \in I^{\prime}$ always commute with the roots $\alpha_{j}$ which appear in these calculations. Note also that for the exceptional set $I^{*}=\mathbb{Z} / n \mathbb{Z}-\{1\}$ which appears in the third case above, the table is to be interpreted as the following equality:

$$
\phi_{0}\left(A_{I^{*}} \alpha_{1}\right)=-2 A_{I^{*}-\{0\}}-\sum_{a \in I^{*} ; a \neq 0} A_{I^{*}-\{a\}} .
$$


One observes that the terms marked (A) or (B) or (C) when grouped together cancel out. We have the following: (A) corresponds to subsets $J$ of size $i-1$ such that $J$ contains neither 1 nor 0 ; and (B) corresponds to subsets $J$ of size $i-1$ such that $J$ contains 0 but not 1 ; and (C) corresponds to subsets $J$ of size $i-1$ such that $J$ contains 1 but not 0 . Every such subset in, say, case (A) will appear in all three case $(\mathrm{A})$ terms. No other subsets (those containing both 0 and 1 ) appear in the sum $\sum_{I} A_{I} \alpha_{1}$.

For example, the element $A_{J}$ with $J=[2,4] \cup[5,7]$ will appear in $\phi_{0}\left(A_{I} \alpha_{1}\right)$ for $I=[2,7]$ or $[1,4] \cup[5,7]$ or $\{0\} \cup[2,4] \cup[5,7]$. The multiplicities will be $-1,2$, and -1 , respectively, which cancel out.

7.2. Proof of Proposition 17.3; Comparison of coproduct. We proceed by first computing directly $\phi_{0} \circ \Delta$ on the generators $h_{i}$ of $\mathbb{B}$.

Lemma 7.7. We have

$$
\phi_{0}\left(\Delta\left(h_{i}\right)\right)=\sum_{0 \leq j \leq i} h_{j} \otimes h_{i-j} .
$$

Proof. In the following computations the indices of roots and the elements $A_{i}$ are to be taken modulo $n$. Let $\beta_{i}=-\alpha_{i}$ be the negative simple roots. We use $\Delta\left(A_{i}\right)=$ $A_{i} \otimes 1+1 \otimes A_{i}+A_{i} \otimes \beta_{i} A_{i}$.

Let $i_{1}, i_{2}, \ldots, i_{l} \in \mathbb{Z} / n \mathbb{Z}$ be a cyclically decreasing sequence. For convenience we assume that if $i_{k}=i_{j}+1$, then $k=j-1$. We have

$$
\begin{gathered}
\Delta\left(A_{i_{1}} A_{i_{2}} \cdots A_{i_{l}}\right)=\prod_{j} \Delta\left(A_{i_{j}}\right) \\
=\left(A_{i_{1}} \otimes 1+1 \otimes A_{i_{1}}+A_{i_{1}} \otimes \beta_{i_{1}} A_{i_{1}}\right) \cdots\left(A_{i_{l}} \otimes 1+1 \otimes A_{i_{l}}+A_{i_{l}} \otimes \beta_{i_{l}} A_{i_{l}}\right)
\end{gathered}
$$

Let us expand the product, by picking one of the three terms in each parentheses. Strictly speaking, we cannot multiply within $\mathbb{A} \otimes_{S} \mathbb{A}$ since it is not a ring. Instead we are calculating the action of $\mathbb{A}$ on $\mathbb{A} \otimes_{S} \mathbb{A}$ via the coproduct: for example $\Delta\left(A_{i} A_{j}\right)=\Delta\left(A_{i}\right) \cdot\left(\Delta\left(A_{j}\right) \cdot(1 \otimes 1)\right) \in \mathbb{A} \otimes_{S} \mathbb{A}$. Formally, for our purposes the calculation proceeds like multiplication.

Because of the cyclically decreasing assumption, the only times we encounter a factor looking like $A_{i_{a}} \beta_{i_{b}}$ (where $\left.a<b\right)$ we have either

$$
A_{i_{a}} \beta_{i_{b}}=\beta_{i_{b}} A_{i_{a}}
$$

or we will have $a=b-1$ and $i_{a+1}=i_{a}-1$ and

$$
A_{i_{a}} \beta_{i_{a}-1}=\left(\beta_{i_{a}-1}+\beta_{i_{a}}\right) A_{i_{a}}+1
$$

If (7.1) ever occurs, then $\beta_{i_{b}}$ commutes with all $A_{i_{c}}$ where $c<b$ and we may ignore the term since eventually we will apply $\phi_{0}$. Similarly, if (17.2) occurs, the contribution of the term involving $\beta_{i_{a}-1}$ is 0 after applying $\phi_{0}$.

Also we perform the calculation

$$
A_{i+1}\left(\beta_{i}\right)^{m}=\beta_{i+1}^{m} A_{i+1}+\beta_{i+1}^{m-1}+\text { other terms, }
$$

where the other terms involve $\beta_{i}$ on the left somewhere (and would be killed by $\phi_{0}$ later).

Let $B$ and $C$ be two subsets of $[0, n-1]$ with total size equal to $i \leq n-1$. We will first describe how to obtain the term $A_{B} \otimes A_{C}$ (which occurs in $h_{|B|} \otimes h_{|C|}$ ) from $\Delta\left(h_{i}\right)$. Define a sequence of integers ("current degree") $(\operatorname{cd}(i): j \in \mathbb{Z} / n \mathbb{Z})$ by $\operatorname{cd}(j)=\max _{0 \leq t \leq n-1}\{|B \cap[j-t, j]|+|C \cap[j-t, j]|-t-1\}$. Since $|B|+|C|<n$, it is not hard to verify that we can find $j$ so that $\operatorname{cd}(j)=0$ and $i \notin B \cup C$. 
We may assume that $j=0$. Let $B=\left\{b_{1}>\cdots>b_{g}>0\right\}$ and $C=\left\{c_{1}>\cdots>\right.$ $\left.c_{h}>0\right\}$. Define a sequence $\left(t_{1}, t_{2}, \ldots, t_{n-1}\right) \in\{L, R, B, E\}^{n-1}$ as follows (where $E=$ empty, $L=$ left,$R=$ right and $B=$ both):

$$
t_{j}= \begin{cases}E & \text { if } \operatorname{cd}(j)=0 \text { and } E \notin B \cup C, \\ L & \text { if } \operatorname{cd}(j)=0 \text { and } E \in B \text { but } E \notin C, \\ R & \text { if } E \notin B \text { and }(\operatorname{cd}(j)>0 \text { or } E \in C), \\ B & \text { otherwise. }\end{cases}
$$

Now let $I=\left\{j \in[1, n-1] \mid t_{j} \neq E\right\} \subset[1, n-1]$. Then $A_{B} \otimes A_{C}$ is obtained from $\Delta\left(A_{I}\right)$ by picking the term $A_{i_{s}} \otimes 1$ if $t_{i_{s}}=L$, the term $1 \otimes A_{i_{s}}$ if $t_{i_{s}}=R$ and $A_{i_{s}} \otimes \beta_{i_{s}} A_{i_{s}}$ if $t_{i_{s}}=B$.

However, when the situation of (7.3) occurs, one has to make a further choice between the two terms. The sequence of integers $(\operatorname{cd}(i))$ tells us the current degree (in the second factor of the tensor product) in $S$ of the term that we want to pick whenever we encounter the situation of (7.3).

For example if $\operatorname{cd}(t)=3$ and $\operatorname{cd}(t+1)=3$, then $t+1 \in B$ or $t+1 \in C$. In the first case we will have $\left(A_{t+1} \otimes 1\right) \cdot\left(a \otimes \beta_{i}^{3} b\right)$, for some $a$ and $b$ not involving $S$, and there is no further choice. In the second case we get

$$
\left(1 \otimes A_{t+1}\right) \cdot\left(a \otimes \beta_{i}^{3} b\right)=a \otimes \beta_{i+1}^{3} A_{t+1} b+a \otimes \beta_{i+1}^{2} b,
$$

modulo terms involving $\beta_{i}$ on the right. One must make a further choice between $\beta_{i+1}^{3} A_{t+1}$ and $\beta_{i+1}^{2}$. We pick the first term since we want $t+1 \in C$ and this agrees with the degree being $\operatorname{cd}(t+1)=3$.

Thus every term of the form $A_{B} \otimes A_{C}$ appears in the expansion of $\phi_{0}\left(\Delta\left(h_{i}\right)\right)$. Conversely, every "current degree" sequence arising from choosing terms in the expansion of $\Delta\left(A_{I}\right)$ as described above corresponds to some pair of subsets $B$ and $C$ with total size $|B|+|C|=|I|$.

Proof of Proposition 7.3. From Lemma 7.7, we have $\Delta_{\mathbb{B}}\left(h_{i}\right)=\phi_{0}\left(\Delta\left(h_{i}\right)\right)$. Now let $a \in \mathbb{B}$ and $b \in \mathbb{B}$ and suppose we have shown that $\Delta_{\mathbb{B}}(a)=\phi_{0}(\Delta(a))$ and $\Delta_{\mathbb{B}}(b)=\phi_{0}(\Delta(b))$. Let $\Delta(a)=\sum_{w, v} A_{w} \otimes a_{w, v} A_{v}$ and $\Delta(b)=\sum_{x, y} A_{x} \otimes b_{x, y} A_{y}$, where $a_{w, v}, b_{x, y} \in S$. Then

$$
\begin{aligned}
\phi_{0}(\Delta(a b)) & =\phi_{0}(\Delta(a) \Delta(b)) \\
& =\phi_{0}\left(\sum_{w, v, x, y} A_{w} A_{x} \otimes a_{w, v} A_{v} b_{x, y} A_{y}\right) \\
& \left.=\sum_{w, v, x, y} A_{w} A_{x} \otimes \phi_{0}\left(a_{w, v}\right) A_{v} \phi_{0}\left(b_{x, y}\right) A_{y} \quad \text { (by Proposition } 7.2\right) \\
& =\phi_{0}(\Delta(a)) \phi_{0}(\Delta(b)) \\
& =\Delta_{\mathbb{B}}(a) \Delta_{\mathbb{B}}(b) \\
& =\Delta_{\mathbb{B}}(a b) .
\end{aligned}
$$

Since the $h_{i}$ generate $\mathbb{B}$, this completes the proof.

\section{Positivity}

The connection between symmetric functions and the geometry of the affine Grassmannian established in Theorem 7.1]allows us to resolve some positivity properties of $k$-Schur functions and affine Schur functions. 
8.1. Positivity of cohomology product. Kumar [16] and Graham [1] have shown that the structure constants of $H^{T}(\mathcal{G} r)$ in the Schubert basis are non-negative polynomials. Thus we have

Corollary 8.1. The product structure constants for the affine Schur functions $\left\{\tilde{F}_{w}(x): w \in W^{0}\right\}$ are non-negative integers, or equivalently the coproduct structure constants for the $k$-Schur functions $\left\{s_{w}^{(k)}(x): w \in W^{0}\right\}$ are non-negative.

The statement for $k$-Schur functions was a conjecture in [21, p. 106].

8.2. Positivity of homology product. Peterson [28] showed that the structure constants of $H_{*}(\mathcal{G} r)$ are special cases of structure constants for the quantum cohomology $Q H^{*}(K / T)$ of the flag manifold. Since these latter structure constants are known to enumerate certain curves in $K / T$, they are necessarily non-negative. Thus we have

Corollary 8.2. The coproduct structure constants for the affine Schur functions $\left\{\tilde{F}_{w}(x): w \in W^{0}\right\}$ are non-negative integers, or equivalently the product structure constants for the $k$-Schur functions $\left\{s_{w}^{(k)}(x): w \in W^{0}\right\}$ are non-negative.

Evidence for Corollary 8.2 had recently been given in 24. It was shown directly and combinatorially that the structure constants for $k$-Schur functions included as a special case the Gromov-Witten invariants of the Grassmannian.

A special case of the homology structure constants can be described rather simply and explicitly.

Corollary 8.3. Let $t_{\lambda}$ be a translation by an antidominant co-root $\lambda \in Q^{-}$. Then

$$
s_{t_{\lambda}}^{(k)}(x) s_{x}^{(k)}(x)=s_{x t_{\lambda}}^{(k)}(x) .
$$

Proof. Combine Proposition 4.5 with Theorem 7.1

If we translate the labeling of $k$-Schur functions from affine Grassmannian permutations to partitions (see [17]), we obtain the following result.

Corollary 8.4. Let $\mu$ be a partition with no part greater than $k(=n-1)$, and let $R$ be a rectangle of the form $R=\left(a^{n-a}\right)$. Then

$$
s_{\rho}^{(k)}(x)=s_{R}^{(k)}(x) s_{\mu}^{(k)}(x),
$$

where $\rho=R \cup \mu$.

A combinatorial proof of Corollary 8.4 was given in [23, Theorem 40]. Peter Magyar communicates to us that he has a purely geometric explanation of Corollary 8.3 .

In fact the homology structure constants are special cases of the coefficients $b_{w, v}$ occurring in the expansion of an affine Stanley symmetric $\tilde{F}_{w}(x)$ in terms of affine Schur functions, as explained in [17. By Proposition 6.7, the $b_{w, v}$ also occur in expressing $s_{w}^{(k)}=\phi_{0}\left(j\left(\sigma_{(w)}\right)\right)$ in the $A_{v}$ basis. Peterson [28] also showed that these coefficients were positive, and thus this resolves the combinatorial Conjecture 38 of [17].

Corollary 8.5. The affine Stanley symmetric functions $\tilde{F}_{w}(x)$ expand positively in terms of affine Schur functions. 
In the special case that $w$ is 321-avoiding, we obtain the positivity of the cylindric Schur functions studied by Postnikov [29] in terms of affine Schur functions, as explained in [17. As a special case we obtain the positive expansion of usual Stanley symmetric functions in terms of Schur functions, first shown in [5, 26]. A combinatorial algorithm intended to give a direct proof of Corollary 8.5 was developed in [20].

Remark 8.6. There is a more direct geometric interpretation of the coefficients $b_{w, v}$. Consider the map $p: \mathcal{G} r \rightarrow \mathcal{G} / \mathcal{B}$ given by the composition $\Omega K \hookrightarrow L K \rightarrow L K / T$. Then we have $p^{*}\left(\sigma_{\mathbb{B}}^{v}\right)=\sum_{v \in W^{0}} b_{w, v} \sigma^{w}$. In other words, under the isomorphism of Theorem 7.1 the affine Stanley symmetric functions $\tilde{F}_{w}(x)$ are the pullbacks $p^{*}\left(\sigma_{\mathbb{B}}^{v}\right)$. This also gives a direct geometric explanation of Stanley symmetric functions.

8.3. Other positivity properties. The $k$-Schur functions are known or conjectured to have other positivity properties which may have interesting geometric explanations. As explained in Section 6.2, the Macdonald polynomials are conjectured to be positive in the $k$-Schur basis, and this suggests a precise connection between the homology of $\mathcal{G} r$ and Cherednik algebras. The $k$-Schur functions are also conjectured to be $(k+1)$-Schur positive, which suggests positivity properties for Schubert classes under the embedding $\Omega S U(n) \hookrightarrow \Omega S U(n+1)$. Finally, the original $t$-analogue $s^{(k)}(x ; t)$, conjectured to be a $\mathbb{N}[t]$-combination of Schur functions, suggests that there may be an additional $\mathbb{C}^{*}$-equivariance to be considered.

\section{Further DireCtions}

There are many potential generalizations of this work, as for the usual Schubert polynomials [25], one may generalize in the $K$-theoretic, quantum, equivariant and other Lie type directions. We note here that the $K$-theoretic version of the nilHecke ring was developed in [15] and a $K$-theoretic version of the affine Schur functions (called affine stable Grothendieck polynomials) was defined in [17]. Also note that combinatorial proofs of the positivity and other theorems, including Corollaries 8.1 and 8.5 are still missing.

It would also be interesting to compare our work with the viewpoint taken in geometric representation theory, for example via the geometric Satake isomorphism. In particular, Ginzburg [10] gives a description of the cohomology of $\mathcal{G} r$ as the symmetric algebra $U\left(\check{\mathfrak{g}}^{e}\right)$ on the centralizer of a principal nilpotent $e$ in the Langlands dual Lie algebra $\mathfrak{\mathfrak { g }}$. The homology Schubert classes corresponding to $G(\mathbf{O})$-orbits, that is, those of the form $\sigma_{t_{\lambda}}$ where $\lambda \in Q^{-}$is anti-dominant, are described explicitly in [10] as matrix coefficients on $\check{\mathfrak{g}}^{e}$. It would be interesting to compare this formula with the $k$-Schur functions.

As evidence for a possible connection, we note that by Proposition 4.5 we have $j\left(\sigma_{\left(t_{\lambda}\right)}\right)=\sum_{w \in W / W_{\lambda}} A_{t_{w \cdot \lambda}}$, and one can compute directly that this element lies not only in the centralizer $Z_{\mathbb{A}_{\text {aff }}}(S)$ but also in the center $Z\left(\mathbb{A}_{\text {aff }}\right)$ of $\mathbb{A}_{\text {aff }}$. It seems natural to ask whether the center $Z\left(\mathbb{A}_{\mathrm{aff}}\right)$ is generated by these elements and the $W_{\text {aff-invariants }} S^{W_{\text {aff }}}$. As the affine nilHecke ring is in a loose sense a deformation of the affine Hecke algebra, this compares well with the identification of the spherical Hecke algebra as the center of the affine Hecke algebra; see [8]. We can show that the center of the affine nilCoxeter algebra is indeed generated by the elements $j\left(\sigma_{\left(t_{\lambda}\right)}\right)$. 
Proposition 9.1. The center of the affine nilCoxeter algebra $\mathbb{A}_{0}$ (for any Lie type) is spanned by the elements

$$
\sum_{w \in W / W_{\lambda}} A_{t_{w \cdot \lambda}}
$$

where $W_{\lambda} \subset W$ is the stabilizer of $\lambda \in Q^{\vee}$.

Proof. We use the length formula (2.1) repeatedly.

Let $a \in Z\left(\mathbb{A}_{0}\right)$ lie in the center. We may assume that $a$ is homogeneous. Suppose first that the coefficient of $A_{w t_{g}}$ is non-zero for some $w \neq$ id. Pick a regular element $h \in Q^{\vee}$ so that the product $w t_{g} t_{h}$ is length adding; that is, $\ell\left(w t_{g} t_{h}\right)=\ell\left(w t_{g}\right)+\ell\left(t_{h}\right)$ so that $A_{w t_{g}} A_{t_{h}}=A_{w t_{g+h}}$. But $A_{t_{h}} A_{w t_{g}}$ is either 0 or equal to $A_{w t_{w h+g}}$. Since $w \cdot h \neq h$, picking $h$ sufficiently large, we can ensure that the coefficient of $A_{w t_{g+h}}$ in $A_{t_{h}} \cdot a$ is 0 . This shows that no term of the form $A_{w t_{g}}$ for $w \neq$ id is present.

Now suppose that $A_{t_{h}}$ occurs in $a$. Suppose $r_{i} \cdot h \neq h$. Then exactly one of $r_{i} t_{h}$ and $t_{h} r_{i}$ has length $\ell\left(t_{h}\right)+1$. Comparing $A_{i} \cdot a$ with $a \cdot A_{i}$, we deduce that the coefficient of $A_{t_{h}}$ is equal to the coefficient of $A_{t_{r_{i} \cdot h}}$ in $a$.

\section{ACKNOWLEDGMENTS}

I am indebted to Luc Lapointe, Jennifer Morse and Mark Shimozono, with whom I have studied $k$-Schur functions. I began working on $k$-Schur and dual $k$-Schur functions more than a year ago when Jennifer first introduced them to me and Mark explained his geometric conjectures to me. I would also like to thank Shrawan Kumar for comments on an earlier version of this paper.

\section{REFERENCES}

1. A. Arabia: T-équivariante de la variété de drapeaux d'un groupe de Kac-Moody, Bull. Soc. Math. France 117 (1989), no. 2, 129-165. MR1015806 (90i:32042)

2. I.N. Bernstein, I.M. Gelfand, and S.I. Gelfand: Schubert cells and cohomology of the spaces $G / P$, Russ. Math. Surv. 28 (1973), 1-26. MR0429933(55:2941)

3. R. Bezrukavnikov, M. Finkelberg, and I. Mirković: Equivariant homology and $K$-theory of affine Grassmannians and Toda lattices, Compos. Math. 141 (2005), no. 3, 746-768. MR2135527 (2006e:19005)

4. R. BотT: The space of loops on a Lie group, Michigan Math. J. 5 (1958), 35-61. MR.0102803 $(21: 1589)$

5. P. Edelman And C. Greene: Balanced tableaux, Adv. Math. 631 (1987), 42-99. MR0871081 (88b:05012)

6. S. Fomin and C. Greene: Noncommutative Schur functions and their applications, Discrete Mathematics 193 (1998), 179-200. MR.1661368(2000c:05149)

7. S. Fomin and R. Stanley: Schubert polynomials and the nilCoxeter algebra, Adv. Math. 103 (1994), 196-207. MR.1265793 (95f:05115)

8. D. Gaitsgory: Construction of central elements in the affine Hecke algebra via nearby cycles, Invent. Math. 144 (2001), 253-280. MR1826370(2002d:14072)

9. H. Garland and M. S. Raghunathan: A Bruhat decomposition for the loop space of a compact group: a new approach to results of Bott, Proc. Nat. Acad. Sci. U.S.A. 72 (1975), no. 12, 4716-4717. MR0417333 (54:5389)

10. V. GinzBurg: Perverse sheaves on a loop group and Langlands' duality, preprint; math. AG/9511007.

11. W. Graham: Positivity in equivariant Schubert calculus, Duke Math. J. 109, no. 3 (2001), 599-614. MR1853356 (2002h:14083)

12. M. Haiman: Hilbert schemes, polygraphs, and the Macdonald positivity conjecture, J. Amer. Math. Soc. 14 (2001), 941-1006. MR1839919 (2002c:14008)

13. J. Humphreys: Reflection groups and Coxeter groups, Cambridge Studies in Advanced Mathematics 29, Cambridge University Press, Cambridge, 1990. MR.1066460 (92h:20002) 
14. B. Kostant and S. Kumar: The nil Hecke ring and the cohomology of $G / P$ for a Kac-Moody group $G$, Adv. in Math. 62 (1986), 187-237. MR0866159 (88b:17025b)

15. B. Kostant and S. Kumar: $T$-equivariant $K$-theory of generalized flag varieties, Proc. Natl. Acad. Sci. USA 84 (1987), 4351-4354. MR0894705 (88m:22048)

16. S. KumAR: Kac-Moody groups, their flag varieties and representation theory, Progress in Mathematics 204, Birkhäuser Boston, Inc., Boston, MA, 2002. MR1923198 (2003k:22022)

17. T. LAm: Affine Stanley symmetric functions, Amer. J. Math., to appear; math.C0/0501335.

18. T. LAM: Schubert polynomials for the affine Grassmannian (extended abstract), Proc. FPSAC, 2006, San Diego.

19. T. Lam, L. Lapointe, J. Morse, and M. Shimozono: Affine insertion and Pieri rules for the affine Grassmannian, preprint, 2006; math.C0/0609110.

20. T. Lam And M. Shimozono: A Little bijection for affine Stanley symmetric functions, preprint, 2006; math.CO/0601483.

21. L. Lapointe, A. Lascoux, And J. Morse: Tableau atoms and a new Macdonald positivity conjecture, Duke Math. J. 116(1) (2003), 103-146. MR.1950481(2004c:05208)

22. L. Lapointe And J. Morse: Schur function analogs for a filtration of the symmetric function space, J. Combin. Theory Ser. A 101(2) (2003), 191-224. MR1961543 (2004c:05209)

23. L. LAPOINTE AND J. MORSE: A $k$-tableaux characterization of $k$-Schur functions, preprint, 2005; math.CO/0505519.

24. L. Lapointe And J. Morse: Quantum cohomology and the $k$-Schur basis, Tran. Amer. Math. Soc., to appear; math. CO/0501529.

25. A. Lascoux and M. Schützenberger: Polynômes de Schubert, C.R. Acad. Sci. Paris, 294 (1982), 447-450. MR0660739 (83e:14039)

26. A. Lascoux and M. SchÜtzenberger: Schubert polynomials and the Littlewood-Richardson rule, Lett. Math. Phys. 10(2-3) (1985), 111-124. MR0815233 (87g:20021)

27. I. G. Macdonald: Symmmetric Functions and Hall Polynomials, Oxford, 1970. MR0553598 (84g:05003)

28. D. Peterson: Lecture notes at MIT, 1997.

29. A. Postnikov: Affine approach to quantum Schubert calculus, Duke Math. J. 128(3) (2005), 473-509. MR2145741 (2006e:05182)

30. A. Pressley and G. Segal: Loop groups, Clarendon Press, Oxford, 1986. MR0900587 (88i:22049)

Department of Mathematics, Harvard University, Cambridge, Massachusetts 02138

E-mail address: tfylam@math.harvard.edu

$U R L:$ http://www.math.harvard.edu/ tfylam 